\title{
Zur Theorie der linearen Differentialgleichungen mit veränderlichen Coefficienten.
}

(Ergänzungen zu der im $66^{\text {sten }}$ Bande dieses Journals enthaltenen

\author{
Abhandlung.) \\ (Von Herrn L. Fuchs.)
}

E in le i t u n g.

Im Folgenden erlaube ich mir einige Ergänzungen zu meiner im $66^{\text {sten }}$ Bande dieses Journals enthaltenen Arbeit zu liefern, und daran einige weitere Entwickelungen zu knüpfen. Die Sätze der $\$ \$$. 4, 5, 6 der angeführten $\mathrm{Ab}-$ handlung ergeben sich aus der Untersuchung beliebiger linearer homogener Differentialgleichungen, eingeschränkt auf das Gebiet eines singulären Punktes, in welchem die Coefficienten eine gewisse Gestalt annehmen. Die bei dieser Untersuchung sich ergebenden Sätze stellen sich nicht bloss als die wahre Quelle der dortigen Entwickelungen dar, sondern gestatten auch anderweitige Anwendungen. Die bei der besonderen Form der Coefficienten aus denselben auf rationale Weise abzuleitende Gleichung wird mit der „Fundamentalgleichung", welche aus den Coefficienten der Differentialgleichung auf transscendente Weise gebildet ist, in engere Beziehung gesetzt, indem die Wurzeln der ersteren in Gruppen vertheilt werden, welche Gruppen einander gleicher Wurzeln der letzteren entsprechen. Die diesen Gruppen zugehörigen Integrale verlaufen in der Umgebung des singulären Punktes gewissermassen für sich selbst, so dass die abgesonderte Betrachtung derselben zu den Fundamentaleigenschaften der Integrale führt, ein Umstand, der sich schon bei der Untersuchung der allgemeinen Form der Integrale in der Umgebung eines beliebigen singulären Punktes in der oben angeführten Abhandlung $\$ .3$ kundgegeben. -

Es wird gezeigt, dass die linearen nicht homogenen Differentialgleichungen sich unserer Untersuchung unterwerfen lassen, wenn man ihnen gewisse lineare homogene Differentialgleichungen zuordnet. Die sich dabei ergebenden Sätze liefern auch ein wahres Kriterium dafür, ob für singuläre Punkte, in deren Umgebung die Coefficienten die oben angeführte besondere Form haben, in den Integralen einer Gruppe Logarithmen auftreten. Dieses bildet alsdann 
den Ausgangspunkt zur Unterscheidung der wirklich singulären Punkte von den nur scheinbaren einer beliebigen Differentialgleichung.

Bei den Citaten aus der oben angeführten Abhandlung möge das Zeichen A. gebraucht werden.

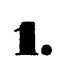

In der eben erwähnten Abhandlung $\$$. 3 ist die Gestalt der Integrale der linearen Differentialgleichung

$$
\text { (1.) } \quad \frac{d^{m} y}{d x^{m}}+p_{1} \frac{d^{m-1} y}{d x^{m-1}}+p_{2} \frac{d^{m-2} y}{d x^{m-2}}+\cdots+p_{m} y=0
$$

in der Umgebung eines singulären Punktes fixirt worden, in der Voraussetzung, dass die Coefficienten der Differentialgleichung in der Umgebung desselben Punktes eindeutig sind. Ist $a$ ein solcher singulärer Punkt, so gehört zu demselben eine Gleichung $\boldsymbol{A}=0$ des $\boldsymbol{m}^{\text {ten }}$ Grades, deren Coefficienten gewisse von der Natur der Differentialgleichung abhängige Constanten sind, welche wir Fundamentalgleichung genannt haben (A. \$. 3 Gl. (6.)). Die Wurzeln derselben $\omega_{1}, \omega_{2}, \ldots \omega_{m}$ sind stets von Null verschieden. Sind nicht zwei derselben gleich, so ist gezeigt worden, dass es ein Fundamentalsystem von Integralen $y_{1}, y_{2}, \ldots y_{m}$ giebt von der Beschaffenheit, dass

$$
\text { (2.) } y_{\mathfrak{a}}=(x-a)^{k} \mathfrak{a} \varphi_{\mathfrak{a}} \quad(\mathfrak{a}=1,2, \ldots m),
$$

wo $k_{\mathfrak{a}}=\frac{1}{2 \pi i} \log \omega_{\mathfrak{a}}$ und $\varphi_{\mathfrak{a}}$ eine in der Umgebung von $a$ eindeutige Function ist. - Wenn hingegen die Fundamentalgleichung mehrfache Wurzeln enthält, wenn z. B. $\omega_{1}$ eine $\lambda$ fache Wurzel derselben ist, so entspricht dieser Gruppe einander gleicher Wurzeln die Integralgruppe

$$
\left(2^{b} .\right) \quad u_{\mathfrak{a}}=(x-a)^{k}{ }_{\mathfrak{a}} \sum_{\mathfrak{l}}^{\mathfrak{a}} \varphi_{\mathfrak{a b}}[\log (x-a)]^{\mathfrak{b}-1} \quad(\mathfrak{a}=1,2, \ldots \lambda),
$$

wo $\varphi_{\mathfrak{a} b}$ in der Ungebung von $a$ eindeutige Functionen von der Beschaffenheit sind, dass $\varphi_{\mathfrak{a b}}$ als eine lineare homogene Function mit constanten Coefficienten von $\varphi_{11}, \varphi_{21}, \ldots \varphi_{a 1}$ dargestellt werden kann, während die Grössen $k_{1}, k_{2}, \ldots k_{\lambda}$ nur um ganze Zahlen differirende Werthe des Ausdruckes $\frac{1}{2 \pi i} \cdot \log \omega_{1}$ bedeuten. Die zu den verschiedenen Wurzelgruppen gehörigen Integralgruppen bilden zusammen ein Fundamentalsystem. Es ist daselbst ein Verfahren angedeutet worden, die lineare Abhängigkeit der Grössen $\varphi_{\mathfrak{a b}}$ näher zu bestimmen. Haben nämlich die Grössen $\omega_{\mathfrak{a b}}$ dieselbe Bedeutung wie in $\mathbf{A} . \$ 3.3 \mathrm{Gl}$. (10.), so hat 
man.das System von Gleichungen:

$$
\left\{\begin{array}{l}
\omega_{1} \sum_{2}^{\mathfrak{a}} \sum_{\mathfrak{b}}(2 \pi i)^{\mathfrak{b}-1} \varphi_{\mathfrak{a} \mathfrak{b}}=\sum_{1}^{\mathfrak{a}-1} \omega_{\mathfrak{a} \mathfrak{b}} \varphi_{\mathfrak{b} 1}, \\
\omega_{1} \sum_{\mathfrak{b}}^{\mathfrak{a}}(\mathfrak{b}-1)(2 \pi i)^{\mathfrak{b}-2} \varphi_{\mathfrak{a} \mathfrak{b}}=\sum_{2}^{\mathfrak{a}-1} \omega_{\mathfrak{b}} \varphi_{\mathfrak{b} 2}, \\
\omega_{1} \sum_{4}^{\mathfrak{a}} \frac{(\mathfrak{b}-1)(\mathfrak{b}-2)}{2}(2 \pi i)^{\mathfrak{b}-3} \varphi_{\mathfrak{a} \mathfrak{b}}=\sum_{\mathfrak{3}}^{\mathfrak{a}-1} \omega_{\mathfrak{a} \mathfrak{b}} \varphi_{\mathfrak{b} 3}, \quad \mathfrak{a}=2,3, \ldots \lambda \\
\vdots \\
\omega_{1}(\mathfrak{a}-1) 2 \pi i \varphi_{\mathfrak{a} \mathfrak{a}}=\omega_{\mathfrak{a}, \mathfrak{a}-1} \varphi_{\mathfrak{a}-1, \mathfrak{a}-1},
\end{array}\right.
$$

Aus der letzten ergiebt sich $\varphi_{\mathfrak{a a}}$, aus der vorletzten $\varphi_{\mathfrak{a}, \mathfrak{a}-1}, \mathbf{u}$. s. w., endlich aus der ersten $\varphi_{\mathfrak{u} 2}$ als lineare homogene Function der Grössen $\varphi$, deren erster Index kleiner ist als $\mathfrak{a}$. Ist $\varphi_{\mathfrak{a} a}$ von Null verschieden, so folgt aus der letzten der Gleichungen (3.), dass auch $\varphi_{11}, \varphi_{22}, \varphi_{33}, \ldots \varphi_{\mathfrak{a}-1, \mathfrak{a}-1}$ von Null verschieden sind. Setzt man daher in dieser Gleichung successive $\mathfrak{a}=2,3, \ldots$ and multiplicirt die entstandenen Gleichungen, so erhält man

$$
\text { (4.) }\left(2 \pi i \omega_{1}\right)^{\mathfrak{a}-1} \cdot 1.2 \ldots(\mathfrak{a}-1) \varphi_{\mathfrak{a} \mathfrak{a}}=\omega_{21} \omega_{32} \ldots \omega_{\mathfrak{a}, \mathfrak{a}-1} \cdot \varphi_{11}, \quad \text { d. h. }
$$

I. Der Coefficient von $[\log (x-\mathfrak{a})]^{a-1}$ im Ausdrucke $\left(2^{b}\right.$.) des Elements $u_{\mathfrak{a}}$ ist entweder Null oder bis auf einen constanten Factor das Integral $u_{1}$.

Dieses Resultat lässt sich auch auf andere Weise herleiten, indem man direct den folgenden Satz beweist:

II. Ist $V=v_{1}+v_{1} \log (x-a)+v_{2}[\log (x-a)]^{2}+\cdots+v_{r}[\log (x-a)]^{r}$ ein Integral der Differentialgleichung (1.), und sind die Coefficienten $v$ bis auf einen allen gemeinschaftlichen Factor $(x-a)^{\alpha}$ in der Umgebung von a eindeutige Functionen, so ist auch der Coefficient der, höchsten Potenz von $\log (x-a)$ ein Integral derselben Differentialgleichung.

Substituirt man nämlich in der Differentialgleichung (1.) $V$ für $y$, ordnet den erhaltenen Ausdruck nach Potenzen von $\log (x-a)$ und dividirt durch $(x-a)^{\alpha}$, so erhält man eine Gleichung der Form:

(5.) $\quad P_{0}+P_{1} \log (x-a)+P_{2}[\log (x-a)]^{2}+\cdots+P_{r}[\log (x-a)]^{r}=0$,

wo $P_{0}, P_{1}, P_{2}, \ldots P_{r}$ in der Umgebung von $a$ eindeutige Functionen sind. Eine Gleichung dieser Form kann aber nur bestehen, wenn die Coefficienten der Potenzen von $\log (x-a)$ einzeln verschwinden.

Denn da sie auch bestehen bleiben muss, wenn $x$ eine beliebige Anzahl von Umläufen um den singulären Punkt $a$ gemacht hat, so folgt: (6.) $P_{0}+P_{1}[\log (x-a)+2 k \pi i]+P_{2}[\log (x-a)+2 k \pi i]^{2}+\cdots+P_{r}[\log (x-a)+2 k \pi i]^{r}=0$ 
für jeden ganzzahligen Werth von $k$. Es entsprächen demnach einem bestimmten Werthe von $x$ unzählig viele Wurzeln $z$ der Gleichung

$$
P_{0}+P_{1} z+P_{2} z^{2}+\cdots+P_{r} z^{r}=0
$$

woraus bekanntlich folgt, dass die Coefficienten $P_{0}, P_{1}, P_{2}, \ldots P_{r}$ verschwinden. - Bezeichnet man aber die linke Seite der Differentialgleichung (1.) mit $\boldsymbol{D}(y)$, so ist $(\boldsymbol{x}-\boldsymbol{a})^{\alpha} \boldsymbol{P}_{r}=\boldsymbol{D}\left(\boldsymbol{v}_{r}\right)$. Die Gleichung $\boldsymbol{P}_{r}=\mathbf{0}$ drückt also aus, dass $v_{r}$ ein Integral dieser Differentialgleichung ist.

Es ist überdies $P_{\mathfrak{a}}=D\left(v_{\mathfrak{a}}\right)+\varphi_{\mathfrak{a}}, \mathfrak{a}=1,2, \ldots r-1$, wo $\varphi_{\mathfrak{a}}$ eine lineare Function der Grössen $v_{\mathfrak{a}+1}, v_{\mathfrak{a}+2}, \ldots v_{r}$ und ihrer Ableitungen ist. Die Gleichungen $P_{\mathfrak{a}}=0, \mathfrak{a}=0,1,2, \ldots r-1$ sind daher Differentialgleichungen für $v_{r-1}$, $v_{r-2}, \ldots v_{0}$, durch welche man successive diese Functionen ermitteln kann.

Da jedes wie $V$ beschaffene Integral eine lineare homogene Function mit constanten Coefficienten einer Integralgruppe $\left(2^{b}\right.$.) ist, so folgt umgekehrt der Satz II. aus dem Satze I. Jedes nicht wie $V$ beschaffene Integral ist, als lineare homogene Function mit constanten Coefficienten der Integrale (2.) und $\left(2^{b}\right.$.), eine Summe solcher wie $V$ beschaffener Integrale.

Zur Transformation eines Fundamentalsystems dient häufig der folgende Satz:

III. Ist $y_{1}, y_{2}, \ldots y_{m}$ ein willkürliches Fundamentalsystem, und sind $v_{1}, v_{2}, \ldots v_{n}$ lineare homogene Functionen mit constanten Coefficienten von beliebigen $n$ Elementen desselben $y_{1}, y_{2}, \ldots y_{n}$ :

$$
\text { (7.) } \quad v_{\mathfrak{a}}=\sum_{1}^{n} c_{\mathfrak{a b}} \cdot y_{\mathfrak{b}} \quad(\mathfrak{a}=1,2, \ldots n),
$$

derart, dass die Determinante

$$
C=\left|\begin{array}{ccc}
c_{11} & \ldots & c_{1 n} \\
\vdots & & \\
c_{n 1} & \ldots & c_{n n}
\end{array}\right|
$$

nicht verschwindet, so bilden $v_{1}, v_{2}, \ldots v_{n}, y_{n+1}, \ldots y_{m}$ ein Fundamentalsystem.

Denn eine Gleichung

$$
\text { (8.) } \quad c_{1} v_{1}+c_{2} v_{2}+\cdots+c_{n} v_{n}+c_{n+1} y_{n+1}+\cdots+c_{m} y_{m}=0
$$

mit constanten Coefficienten wäre gleichbedeutend mit

$$
y_{1} \sum_{1}^{n} c_{\mathfrak{b}} c_{b 1}+\cdots+y_{n} \sum_{1}^{n} c_{\mathfrak{b}} \cdot c_{\mathfrak{b} n}+c_{n+1} \cdot y_{n+1}+\cdots+c_{m} y_{m}=0
$$

Da aber $y_{1}, y_{2}, \ldots y_{m}$ ein Fundamentalsystem ist, so könnte diese Gleichung nur stattinden, wenn die Coefficienten der verschiedenen $y$ verschwinden. 
Setzt man aber die Coefficienten von $y_{1}, y_{2}, \ldots y_{n}$ der Null gleich, so folgt, dass entweder $c_{1}=c_{2}=\cdots=c_{n}=0$, und daher auch in (8.) $c_{n+1}=\cdots=c_{m}=0$, oder $\boldsymbol{C}=\mathbf{0}$. Diese letztere Gleichung widerspricht aber der Voraussetzung.

Die Bedingung, dass die Determinante $C$ nicht verschwindet, ist offenbar gleichbedeutend mit der Bedingung, dass eine Gleichung der Gestalt:

$$
C_{1} v_{1}+C_{2} v_{2}+\cdots+C_{n} v_{n}=0,
$$

mit constanten Coefficienten nicht stattfindet.

\section{2.}

Es werde in der Differentialgleichung

$$
\frac{d^{m} y}{d x^{m}}+p_{1} \frac{d^{m-1} y}{d x^{m-1}}+p_{2} \frac{d^{m-2} y}{d x^{m-2}}+\cdots+p_{m} y=0
$$

$y=(x-a)^{r} z$ gesetzt, wo $r$ eine beliebige Grösse, so geht dieselbe über in:

$$
\left\{\begin{array}{c}
(x-a)^{m} \frac{d^{m} z}{d x^{m}}+(x-a)^{m-1} P_{1} \frac{d^{m-1} z}{d x^{m-1}}+(x-a)^{m-2} P_{2} \frac{d^{m-2} z}{d x^{m-2}}+\cdots \\
+(x-a) P_{m-1} \frac{d z}{d x}+P_{m} z=0
\end{array}\right.
$$

wo

$$
\text { (3.) }\left\{\begin{array}{c}
P_{\mathfrak{a}}=\sum_{\mathfrak{0}}^{\mathfrak{a}-1} \frac{(m-\mathfrak{b})(m-\mathfrak{b}-1) \ldots(m-\mathfrak{a}+1)}{\mathfrak{a} !} r(r-1) \ldots(r-\mathfrak{a}+\mathfrak{b}+1)(x-a)^{\mathfrak{b}} p_{\mathfrak{b}}+(x-a)^{\mathfrak{a}} p_{\mathfrak{a}} \\
\left(p_{0}=1\right) .
\end{array}\right.
$$

Wenn die Coefficienten $p$ die Form haben $p_{\mathfrak{a}}=q_{\mathfrak{a}}(x-a)^{-\mathfrak{a}}$, wo $q_{\mathfrak{a}}$ in der Umgebung von $a$ eindeutig, continuirlich und endlich, so folgt aus (3.), dass $\boldsymbol{P}_{\mathfrak{a}}$ ebenfalls in der Umgebung von $\boldsymbol{a}$ eindeutig, continuirlich und endlich ist. Da die Differentialgleichung (2.) in die Differentialgleichung (1.) übergeht, wenn man $z=(x-a)^{-r} y$ setzt, so folgt umgekehrt, dass wenn die Grössen $P_{\mathfrak{a}}$ in der Umgebung von $a$ eindeutig, continuirlich und endlich sind, die Ausdrücke $p_{\mathfrak{a}}(x-a)^{\mathfrak{a}}$ dieselbe Eigenschaft haben. Unter derselben Voraussetzung giebt es ein Fundamentalsystem von Integralen der Differentialgleichung (1.) $y_{1}, y_{2}, \ldots y_{m}$, dessen Elemente wie $F$ beschaffene Functionen (A. \$. 6) und resp. zu gewissen Exponenten $r_{1}, r_{2}, \ldots r_{m}$ gehören. Diesem Fundamentalysteme entspricht ein ähnlich beschaffenes Fundamentalsystem der Differentialgleichung (2.) $z_{1}, z_{2}, \ldots z_{m}$, dessen Elemente resp. zu den Exponenten $\varrho_{1}=r_{1}-r, \varrho_{2}=r_{2}-r, \ldots \rho_{m}=r_{m}-r$ gehören. - Wir nehmen $r$ so an, dass die letzteren Exponenten grösser als $m$ sind. 
Bezeichnet man mit $\Delta_{0}$ die Determinante des Fundamentalsystems $y_{1}, y_{2}, \ldots y_{m}$, so ist (A. $\$ .2$ Gl. (3.))

$$
\Delta_{0}=C e^{-\int p_{1} d x} \text {. }
$$

Ist ebenso $\Delta_{0}^{\prime}$ die Determinante des Fundamentalsystems $z_{1}, z_{2}, \ldots z_{m}$, so ist

$$
\Delta_{0}^{\prime}=C^{\prime} e^{-\int \frac{P_{1}}{x-a} d x}
$$

wo $C$ und $C^{\prime}$ nicht verschwindende Constanten bedeuten. Da nun nach (3.) $P_{1}=m r+(x-a) p_{1}$, so folgt

daher

$$
\Delta_{0}^{\prime}=C^{\prime}(x-a)^{-m r} e^{-\int_{p_{1}} d x}
$$

$$
\text { (4.) } \quad \Delta_{0}=C^{\prime \prime}(x-a)^{m r} \Delta_{0}^{\prime} \text {, }
$$

wenn man $\frac{C}{C^{\prime}}=C^{\prime \prime}$ setzt. Es ist von Wichtigkeit nachzuweisen, dass der Ausdruck $D_{0}=A_{0} \cdot(x-a)^{-\Sigma r_{\mathfrak{a}}+\frac{m(m-1)}{2}}$, wo $\Sigma r_{\mathfrak{a}}$ die Summe der Exponenten $r_{1}, r_{2}, \ldots r_{m}$ bedeutet, für $x=a$ nicht verschwindet. Dieses ist (A. \$. 4) mit Hülfe der Productenform von $\Delta_{0}$ geschehen. Allein der dortige Beweis erfordert einige Erörterungen für den Fall, dass einige der Exponenten $r$ kleiner als $m$ und positive ganze Zahlen sind. Um diese zu vermeiden, beweist man nach (A.\$.4) aus der Productform von $\Delta_{0}^{\prime}$, dass $D_{0}^{\prime}=\Delta_{0}^{\prime}(x-a)^{-\Sigma \varrho_{\mathfrak{a}}+\frac{m(m-1)}{2}}$, wo $\Sigma \varrho_{\mathfrak{a}}$ die Summe der Exponenten $\varrho_{1}, \varrho_{2}, \ldots \varrho_{m}$ bedeutet, nicht verschwindet, da wir über diese Exponenten so verfügt haben, dass sie sämmtlich grōsser als $m$ sind. Aus der Gleichung $\Sigma \varrho_{\mathfrak{a}}=-m r+\Sigma r_{\mathfrak{a}}$ und Gleichung (4.) folgt aber $D_{0}=C^{\prime \prime} . D_{0,}^{\prime}$, folglich ist auch $D_{0}$ für $x=a$ von Null verschieden.

\section{3.}

Sind die Coefficienten der Differentialgleichung

$$
\text { (1.) } \frac{d^{m} y}{d x^{m}}+p_{1} \frac{d^{m-1} y}{d x^{m-1}}+p_{2} \frac{d^{m-2} y}{d x^{m-2}}+\cdots+p_{m} y=0
$$

in der Umgebung des singulären Punktes $a$ eindeutig, und die Integrale für $x=a$ nur so unstetig, dass sie mit bestimmten Potenzen von $(x-a)$ multiplicirt endlich werden, bilden feruer $y_{1}, y_{2}, \ldots y_{m}$ das den Gleichungen (2.) und $\left(2^{b}\right.$.) der $N^{\circ} .1$ entsprechende Fundamentalsystem von Integralen, so sind sie wie $F$ (A. \$. 6) beschaffene Functionen, die resp. zu den Exponenten $r_{1}, r_{2}, \ldots r_{n}$ gehören mögen. Bezeichnet man wieder mit $\Delta_{0}$ die Determinante dieses 
Fundamentalsystems, mit $\Delta_{\mathfrak{a}}$ diejenige Determinante, welche man aus $\Delta_{0}$ erhält, wenn man die $\mathfrak{a}^{\text {te }}$ Verticalreihe durch $\frac{d^{m} y_{1}}{d x^{m}}, \frac{d^{m} y_{2}}{d x^{m}}, \cdots \frac{d^{m} y_{m}}{d x^{m}}$ ersetzt, so ist

$$
\text { (2.) } \quad p_{\mathfrak{a}}=-\frac{\Delta_{\mathfrak{a}}}{\Delta_{0}} \quad \text { (A. } \$ \text {. } 4 \text { Gl. (7.)). }
$$

Nun aber ist (A. \$. 4 II. und III.)

$$
D_{\mathfrak{a}}=\Delta_{\mathfrak{a}}(x-a)^{-\Sigma r_{\mathfrak{a}}+\frac{m(m-1)}{2}+a} \quad(\mathfrak{a}=0,1, \ldots m)
$$

in der Umgebung von $a$ eindeutig, endlich und continuirlich, und (s. die vor. $\mathrm{N}^{\circ}$.) $D_{0}$ für $x=a$ von Null verschieden, folglich ist

$$
p_{\mathfrak{a}}(x-a)^{\mathfrak{a}}=-\frac{D_{\mathfrak{a}}}{D_{0}}
$$

für $x=a$ nicht unendlich. Man hat also den Satz:

Wenn die Integrale einer linearen homogenen Differentialgleichung für einen singulären Punkt a, in dessen Umgebung die Coefficienten derselben eindeutig sind, nur so unstetig werden, dass sie mit bestimmten Potenzen von $(x-a)$ multiplicirt nicht mehr unendlich sind, so hat die Differentialgleichung in der Umgebung des Punktes a die Gestalt:

$$
(x-a)^{m} \frac{d^{m} y}{d x^{m}}+(x-a)^{m-1} P_{1} \frac{d^{m-1} y}{d x^{m-1}}+(x-a)^{m-2} P_{2} \frac{d^{m-2} y}{d x^{m-2}}+\cdots+P_{m} y=0,
$$

wo $P_{1}, P_{2}, \ldots P_{m}$ in der Umgebung des Punktes a eindeutig, continuirlich und endlich sind.

Dieser Satz setzt nichts über die Beschaffenheit der Coefficienten der Differentialgleichung und deren Integrale ausserhalb der Umgebung von $a$ voraus. Man kann aber aus demselben den Satz in No. 4 Gl. (12.) der Abh. herleiten. Sind nämlich die Coefficienten der Differentialgleichung (1.) überall eindeutige Functionen, die in einer endlichen Anzahl von Punkten $a_{1}, a_{2}, \ldots a_{\varphi}$ unstetig werden, und sind die Integrale für jeden dieser singulären Punkte $a_{\lambda}$ nur so unstetig, dass sie mit bestimmten Potenzen von $x-a_{\lambda}$ multiplicirt nicht mehr unendlich werden, so folgt aus dem eben bewiesenen Satze, dass $p_{\mathfrak{a}} . \psi^{\mathfrak{a}}$ für jeden endlichen Werth von $x$ endlich ist, wenn man

$$
\left(x-a_{1}\right)\left(x-a_{2}\right) \ldots\left(x-a_{\rho}\right)=\psi
$$

setzt. Wenn die Integrale ausserdem für $x=\infty$ nur so unstetig werden, dass sie mit bestimmten Potenzen von $x$ multiplicirt nicht mehr unendlich sind, so folgt auf dieselbe Weise wie für die übrigen singulären Punkte, dass $p_{\mathfrak{a}} x^{\mathfrak{a}}$ für $x=\infty$ nicht unendlich ist. Hieraus aber ergiebt sich, wenn man

$$
p_{\mathfrak{a}} \psi^{\mathfrak{a}}=Q_{\mathfrak{a}}
$$


setzt, dass $\frac{Q_{\mathfrak{a}}}{x^{\mathfrak{a}(\rho-1)}}$ für $x=\infty$ nicht unendlich ist. Da also $Q_{\mathfrak{a}}$ eine überall eindeutige, für jeden endlichen Werth endliche Function ist, die für $x=\infty$ höchstens von der Ordnung $\mathfrak{a}(\varrho-1)$ unendlich wird, so ist $Q_{\mathfrak{a}}$ eine ganze rationale Function höchstens vom Grade $\mathfrak{a}(\varrho-1)$. Hiermit ist aber die Form der Differentialgleichung (12.) in No. 4 der A. erwiesen.

\section{4.}

Wir haben (A. \$. 5 u. 6) bewiesen, dass der Differentialgleichung (1.) $(x-a)^{m} \frac{d^{m} y}{d x^{m}}+(x-a)^{m-1} P_{1}(x) \frac{d^{m-1} y}{d x^{m-1}}+(x-a)^{m-2} P_{2}(x) \frac{d^{m-2} y}{d x^{m-2}}+\cdots+P_{m}(x) y=0$, wo $P_{1}(x), P_{2}(x), \ldots P_{m}(x)$ in der Umgebung von $a$ eindeutige, endliche und continuirliche Functionen sind, ein Fundamentalsystem von Integralen $r_{1}, \eta_{2}, \ldots \eta_{m}$ entspricht, dessen Elemente wie $\boldsymbol{F}$ beschaffene Functionen sind, die resp. zu den Exponenten $r_{1}, r_{2}, \ldots r_{m}$ gehören, welche Wurzeln der Gleichung :

$$
\left\{\begin{array}{c}
r(r-1) \ldots(r-m+1)+P_{1}(a) r(r-1) \ldots(r-m+2) \\
+P_{2}(a) r(r-1) \ldots(r-m+3)+\cdots+P_{m}(a)=0 .
\end{array}\right.
$$

Umgekehrt ist der Exponent, zu welchem irgend ein wie $F$ beschaffenes Integral der Differentialgleichung gehört, eine Wurzel der Gleichung (2.). -

Bildet man für die Differentialgleichung (1.) das Fundamentalsystem, welches in No. 1 unter (2.) und $\left(2^{b}.\right)$ angeführt worden, so enthalten die in demselben vorkommenden Functionen $\varphi_{\mathfrak{a}}$ und $\varphi_{\mathfrak{a b}}$ in ihrer Entwickelung nach Potenzen von $x-a$ nur eine endliche Anzahl von Potenzen von $(x-a)^{-1}$; die Elemente des Fundamentalsystems sind daher wie $F$ beschaffene Functionen. Da nun die Grössen $k_{\mathfrak{a}}$ (No. 1) nur bis auf additive ganze Zahlen bestimmt sind, so wählen wir dieselben für die Differentialgleichung (1.) so, dass $k_{\mathfrak{a}}$ der Exponent ist, zu welchem das Element $y_{\mathfrak{a}}$ oder $u_{\mathfrak{a}}$ gehört. Hieraus folgt:

I. Das $\frac{1}{2 \pi i}$ fache des Logarithmus jeder Wurzel der der Differentialgleichung (1.) zugehörigen Fundamentalgleichung (A. \$. 3 Gl. (6.)) ist bis auf additive ganze Zahlen eine Wurzel der Gleichung (2.).

Ist $\eta$ irgend ein Element des Fundamentalsystems $\eta_{1}, \eta_{2}, \ldots \eta_{m}$, welches zum Exponenten $r$ gehört, so ist

$$
\eta(x-a)^{-r}=\psi_{1}+\psi_{2} \log (x-a)+\psi_{3}[\log (x-a)]^{2}+\cdots+\psi_{\lambda}[\log (x-a)]^{\lambda-1},
$$

wo $\psi_{1}, \psi_{2}, \ldots \psi_{2}$ in der Umgebung von $a$ eindeutig, continuirlich und endlich 
sind. Daher kann die lineare homogene Function der Elemente des Fundamentalsystems (2.) und $\left(2^{b}\right.$.) der No. 1 mit constanten Coefficienten, durch welche $\eta$ dargestellt wird, nur diejenigen Elemente dieses Fundamentalsystems enthalten, deren zugehörige Exponenten sich von $r$ nur um ganze Zahlen unterscheiden, also die Elemente einer Gruppe $\left(2^{b}\right.$.) in No. 1. Hieraus folgt:

II. Das 2лifache jeder Wurzel der Gleichung (2.) ist der Logarithmus einer Wurzel der der Differentialgleichung (1.) zugehörigen Fundamentalgleichung (A. \$. 3 Gl. (6.)).

Aus dem Satze I. folgt, dass verschiedenen Wurzeln der Fundamentalgleichung solche Wurzeln der Gleichung (2.) entsprechen, die weder gleich, noch um ganze Zahlen von einander verschieden sind. Umgekehrt folgt daher aus dem Satze II., dass jeder Gruppe von Wurzeln der Gleichung (2.), die entweder gleich, oder um ganze Zahlen von einander verschieden sind, eine Gruppe gleichvieler einander gleicher Wurzeln der Fundamentalgleichung entspricht. Es sei eine solche Gruppe von Wurzeln der Gleichung (2.): $r_{1}, r_{2}, \ldots r_{i}=(R)$, so geordnet, dass der reelle Theil irgend einer derselben nicht kleiner ist als der reelle Theil irgend einer nachfolgenden, so giebt es ein Integral $v_{1}$, der Gestalt $v_{1}=(x-a)^{r_{1}} \varphi$, wo $\varphi$ in der Umgebung von $a$ eindeutig, continuirlich und endlich und in $a$ von Null verschieden ist. Der Beweis hierfür ist genau übereinstimmend mit dem Beweise des Satzes, dass ein derartiges Integral derjenigen Wurzel der Gleichung (2.) zugehört, deren reeller Theil nicht kleiner ist, als der reelle Theil irgend einer anderen Wurzel (A. \$.5). Denn es genügt die hier über $r_{1}$ gemachte Voraussetzung, um das, worauf es bei diesem Beweise ankommt, festzustellen, dass nämlich der Coefficient von $c_{k+1}$ in Gl. (5.) der A. $\$$. 5 für keinen ganzzahligen positiven Werth von $k$ verschwindet.

Setzt man in die Differentialgleichung (1.) $y=v_{1} \int z d x$, so gehört der Differentialgleichung für $z$ ein Integral $z_{1}$ an, der Gestalt $z_{1}=(x-a)^{r_{3}-r_{1}-1} \varphi^{\prime}$, wo $\varphi^{\prime}$ dieselben Eigenschaften wie $\varphi$ hat. Setzt man in die letzterhaltene Differentialgleichung $z=z_{1} \int t d x$, so gehört der Differentialgleichung für $t$ ein Integral $t_{1}$ an, der Gestalt $t_{1}=(x-a)^{r_{3}-r_{2}-1} \varphi^{(2)}$, wo $\varphi^{(2)}$ dieselben Eigenschaften wie $\varphi$ hat. Fährt mản so fort, so erhält man eine Differentialgleichung der Ordnung $m-(\lambda-1)$, der ein Integral $w_{1}=(x-a)^{r_{\lambda}-r_{\lambda-1}-1} \varphi^{(\lambda-1)}$ angehört, wo $\varphi^{(\lambda-1)}$ dieselben Eigenschaften hat wie $\varphi$. Alsdann sind $v_{2}=v_{1} \int \dot{z}_{1} d x$, $v_{3}=v_{1} \int z_{1} d x \int t_{1} d x, \ldots v_{\lambda}=v_{1} \int z_{1} d x \int t_{1} d x \ldots \int w_{1} d x$ Integrale der Differen- 
tialgleichung (1.), welche resp. zu den Exponenten $r_{2}, r_{3}, \ldots r_{\lambda}$ gehören (A. $\$$. 5 u. 6 ).

Bezeichnet man diejenige Function, in welche eine beliebige Function $f(x)$ nach einem Umlaufe um $a$ übergeht, mit $[f(x)]^{\prime}$, so ist von einer Function $F$, wie sie in der A. \$. 6 charakterisirt ist, ersichtlich, dass sie die Eigenschaften

$$
\frac{d}{d x}[F]^{\prime}=\left[\frac{d F}{d x}\right]^{\prime} \text { und } \int[F]^{\prime} d x=\left[\int F d x\right]^{\prime}+\text { Const. }
$$

besitzt. Da aber $v_{1}, z_{1}, t_{1}, \ldots w_{1}, v_{2}, v_{3}, \ldots v_{\lambda}$, wie $F$ beschaffene Functionen (A. \$. 6) und ausserdem $v_{1}(x-a)^{-r_{1}} z_{1}, t_{1}, \ldots w_{1}$ in der Umgebung von $a$ eindeutig sind, so folgt, dass auch $\frac{v_{2}}{v_{1}}, \frac{v_{3}}{v_{1}}, \ldots$ wie $F$ beschaffene Functionen sind und daher

$$
\left[\frac{d}{d x} \frac{v_{2}}{v_{1}}\right]^{\prime}=\frac{d}{d x}\left[\frac{v_{2}}{v_{1}}\right]^{\prime}=\frac{d}{d x} \frac{v_{2}}{v_{1}} \text {. }
$$

Durch Integration ergiebt sich

$$
\left[\frac{v_{2}}{v_{1}}\right]^{\prime}=\frac{v_{2}}{v_{1}}+C
$$

wo $C$ eine Constante bedeutet.

Entspricht nun der Gruppe $(R)$ die Gruppe $(\boldsymbol{W})$ der gleichen Wurzeln der Fundamentalgleichung: $\omega_{1}=\omega_{2}=\cdots=\omega_{\lambda}$, so ist $v_{1}^{\prime}=\omega_{1} v_{1}$. Daher ergiebt die letzte Gleichung:

$$
\text { (3.) }\left[v_{2}\right]^{\prime}=\omega_{21} v_{1}+\omega_{1} v_{2} \text {, }
$$

wenn man $C \omega_{1}=\omega_{21}$ setzt. Ferner ist

$$
\begin{gathered}
{\left[\frac{d}{d x} \frac{v_{3}}{v_{1}}\right]^{\prime}=\frac{d}{d x}\left[\frac{v_{3}}{v_{1}}\right]^{\prime}=\left[z_{1} \int t_{1} d x\right]^{\prime}=z_{1}\left[\int t_{1} d x\right]^{\prime}=z_{1}\left\{\int\left[t_{1}\right]^{\prime} d x+C_{1}\right\}} \\
=z_{1}\left\{\int t_{1} d x+C_{1}\right\}=z_{1} \int t_{1} d x+C_{1} z_{1},
\end{gathered}
$$

wo $C_{1}$ eine neue Constante. Durch Integration folgt:

$$
\left[\frac{v_{3}}{v_{1}}\right]^{\prime}=C_{1} \int z_{1} d x+\int z_{1} d x \int t_{1} d x+C_{2},
$$

wo $C_{2}$ eine fernere Constante. Daher ist

$$
\text { (4.) }\left[v_{3}\right]^{\prime}=\omega_{31} v_{1}+\omega_{32} v_{2}+\omega_{1} v_{3} \text {, }
$$

wenn man $C_{2} \omega_{1}=\omega_{31}, C_{1} \omega_{1}=\omega_{32}$ setzt. So fortfahrend findet man

$$
\left\{\begin{array}{c}
{\left[v_{4}\right]^{\prime}=\omega_{41} v_{1}+\omega_{42} v_{2}+\omega_{43} v_{3}+\omega_{1} v_{4}} \\
\vdots \\
{\left[v_{\lambda}\right]^{\prime}=\omega_{\lambda 1} v_{1}+\omega_{\lambda 2} v_{2}+\cdots+\omega_{1} v_{\lambda} .}
\end{array}\right.
$$


Vergleicht man die Gleichungen (3.), (4.), (5.) mit den Gleichungen (10.) (A. \$. 3), so folgt, dass man die Integralgruppe $v_{1}, v_{2}, \ldots v_{\lambda}$ mit derjenigen der $u_{1}, u_{2}, \ldots u_{\lambda}\left(\mathrm{N}^{\circ} .1\left(2^{b}.\right)\right)$, welche der Gruppe $(W)$ der Wurzeln der Fundamentalgleichung entspricht, identificiren kann. Hierdurch sind aber die Exponenten $k_{1}, k_{2}, \ldots k_{\lambda}$ bestimmt, derart, dass $k_{1}=r_{1}, k_{2}=r_{2}, \ldots k_{\lambda}=r_{\lambda}$. Zwischen den Integralen $v_{1}, v_{2}, \ldots v_{\lambda}$ findet keine lineare homogene Relation mit constanten Coefficienten statt. Dieses folgt ebenso wie in A. $\$$. 2 Gl.(8.). -

Fasst man das Vorhergehende zusammen, so ergiebt sich

III. Vertheilt man die Wurzeln der Gleichung (2.) in Gruppen $(\boldsymbol{R})$ derart, dass jede Gruppe einander gleiche oder von einander um ganze Zahlen verschiedene Wurzeln enthält, so entspricht jeder dieser Gruppen eine Gruppe $(W)$ gleich vieler einander gleicher Wurzeln der Fundamentalgleichung. Das 2лifache der Wurzeln einer Gruppe $(R)$ ist ein Logarithmus der Wurzel der entsprechenden Gruppe $(\boldsymbol{W})$.

Ferner:

IV. Jeder Gruppe $(\boldsymbol{R})$ mit den Wurzeln $r_{1}, r_{2}, \ldots r_{\lambda}$ entspricht eine Gruppe von wie $F$ beschaffenen Integralen $u_{1}, u_{2}, \ldots u_{\lambda}$ derart, dass $u_{\mathfrak{a}}$ zum Exponenten $r_{\mathfrak{a}}$ gehört. Sind die Wurzeln $r$ so geordnet, dass der reelle Theil irgend einer derselben nicht kleiner ist als der reelle Theil irgend einer nachfolgenden, und ist $\omega$ eine der gleichen Wurzeln der dieser Gruppe entsprechenden Gruppe $(W)$, so ist es möglich das System der Integrale $u_{1}, u_{2}, \ldots u_{\lambda}$ so zu gestalten, dass

$$
\text { (6.) }\left[u_{\mathfrak{a}}\right]^{\prime}=\omega_{\mathfrak{a} 1} u_{1}+\omega_{\mathfrak{a} 2} u_{2}+\cdots+\omega_{\mathfrak{a} a-1} u_{\mathfrak{a}-1}+\omega_{1} u_{\mathfrak{a}} \quad(\mathfrak{a}=1,2, \ldots \lambda),
$$

wo $\omega_{\mathfrak{a} 1}, \omega_{\mathfrak{a} 2}, \ldots$ Constanten sind. Die Integrale selbst haben die Gestalt

$$
\text { (7.) } \quad u_{\mathfrak{a}}=(x-a)^{r_{\mathfrak{a}}} \sum_{\mathfrak{l}}^{\mathfrak{a}} \varphi_{\mathfrak{b}}[\log (x-a)]^{\mathfrak{b}-1} \quad(\mathfrak{a}=1,2, \ldots \lambda),
$$

wo $\varphi_{\mathfrak{a} 1}, \ldots \varphi_{\mathfrak{a} \mathfrak{a}}$ in der Umgebung von a endlich, eindeutig und continuirlich und für $x=a$ nicht sämmtlich Null sind. Die Functionen $(x-a)^{r_{\mathfrak{a}}} \varphi_{\mathfrak{a} \mathfrak{b}}(\mathfrak{b}=2, \ldots \lambda)$ lassen sich durch die Functionen $(x-a)^{r_{\mathfrak{a}^{\prime}}} \varphi_{\mathfrak{a}^{\prime} \mathfrak{b}}(\mathfrak{b}=1, \ldots \lambda)$, wo $\mathfrak{a}^{\prime}<\mathfrak{a}$, linear, homogen und mit constanten Coefficienten ausdrücken. Insbesondere ist $\varphi_{\mathfrak{a} \mathfrak{a}}=$ Const. $\varphi_{11}$.

Zwischen den Integralen ein und derselben Gruppe findet keine lineare homogene Relation mit constanten Coefficienten statt; die Gesammtheit der Gruppen bildet ein Fundamentalsystem. 
Aus dem Umstande, dass die Functionen $(x-a)^{r_{\mathfrak{a}}} \varphi_{\mathfrak{a} \mathfrak{b}}(\mathfrak{b}=2, \ldots \lambda)$ sich linear, homogen und mit constanten Coefficienten durch die Functionen $(x-a)^{r_{\mathfrak{a}^{\prime}}} \varphi_{\mathfrak{a}^{\prime} \mathfrak{b}}(\mathfrak{b}=1, \ldots \lambda)$, wo $\mathfrak{a}^{\prime}<\mathfrak{a}$, ausdrücken lassen, folgert man, dass dieselben zu Wurzeln der Gruppe $(\boldsymbol{R})$ als Exponenten gehören, deren Index kleiner ist als $\mathfrak{a}$, deren reeller Theil also grösser ist als der reelle Theil von $r_{\mathfrak{a}}$, wenn nicht Wurzeln derselben Gruppe $(\boldsymbol{R})$ einander gleich sind. Da nun $u_{\mathfrak{a}}$ zum Exponenten $r_{\mathfrak{a}}$ gehört, so gehört nothwendigerweise $\varphi_{\mathfrak{a} 1}(x-a)^{r_{\mathfrak{a}}}$ zum Exponenten $r_{\mathfrak{a}}$, und $\varphi_{\mathfrak{a} 1}$ kann nur verschwinden, wenn Wurzeln derselben Gruppe einander gleich sind, da ausserdem die Integrale (7.) nicht identisch Null sind. Man hat daher den Satz:

V. Sind die Wurzeln einer Gruppe $(\boldsymbol{R})$ von einander verschieden, so fehlt in keinem Elemente der zugehörigen Integralgruppe (7.) das vom Logarithmus freie Glied, und zwar gehört dasselbe zu demjenigen Exponenten, welchem das Integral selbst zugeordnet ist.

Ist $\eta$ ein wie $F$ beschaffenes Integral, so ist dasselbe offenbar eine lineare homogene Function mit constanten Coefficienten nur von denjenigen Elementen des Fundamentalsystems des Satzes IV., welche ein und derselben Wurzelgruppe $(\boldsymbol{R})$ entsprechen, und zwar derjenigen, welche den Exponenten, zu welchem $\eta$ gehört, enthält. Ist derselbe $r_{\beta}$, während $r_{1}, r_{2}, \ldots r_{\beta-1}, r_{\beta+1}, \ldots r_{\lambda}$ die übrigen Wurzeln der Gruppe $(R)$ vorstellen, deren Elemente wie im Satze IV. geordnet sind, sind ferner $u_{1}, u_{2}, \ldots u_{\lambda}$ die der Gruppe $(R)$ entsprechende Integralgruppe (Gl. (7.)), so ist zunächst

(8.) $\eta=c_{1} u_{1}+c_{2} u_{2}+\ldots+c_{\lambda} u_{\lambda}$.

wo $c_{1}, c_{2}, \ldots c_{\lambda}$ Constanten sind. Sind nicht zwei Wurzeln der Gruppe einander gleich, und multiplicirt man diese Gleichung mit $(x-a)^{-r_{2}}$, und setzt $x=a$, so erhält man $0=c_{\lambda} u_{\lambda}(x-a)^{-r_{\lambda}}$ (für $x=a$ ). Da aber der Factor von $c_{\lambda}$ von Null verschieden ist, so folgt, dass $c_{\lambda}$ verschwindet. Dieses vorausgesetzt folgt durch Multiplication der Gleichung (8.) mit $(x-a)^{-r_{\lambda-1}}$ auf dieselbe Weise, dass $c_{\lambda-1}$ verschwindet. Und so fort bis $c_{\beta+1}$. Man hat daher:

$$
\text { (9.) } \eta=c_{1} u_{1}+c_{2} u_{2}+\cdots+c_{\beta} u_{\beta} \text {. D. h. }
$$

VI. Ist $(R)$ diejenige Wurzelgruppe des Satzes IV., welche den Exponenten $r_{\beta}$ enthält, zu dem ein wie $F$ beschaffenes Integral $\eta$ gehört, und sind nicht zwei Wurzeln in $(\boldsymbol{R})$ einander gleich, so ist $\eta$ eine lineare homogene Function mit constanten Coefficienten nur von denjenigen Elementen der der 
Gruppe $(\boldsymbol{R})$ entsprechenden Integralgruppe des Satzes IV., deren zugehörige Exponenten einen nicht kleineren reellen Theil als $r_{\beta}$ haben.

Da die vom Logarithmus freien Glieder in $u_{1}, u_{2}, \ldots u_{\beta}$ sämmtlich von Null verschieden sind und zu verschiedenen Exponenten gehören, so folgt auch :

VII. Sind nicht zwei Wurzeln in $(R)$ einander gleich, so ist der vom Logarithmus freie Theil von $\eta$ von Null verschieden, und gehört mit $\eta$ zu demselben Exponenten.

Da jedes Integral, als lineare homogene Function mit constanten Coefficienten der Elemente des Fundamentalsystems des Satzes IV., eine Summe von wie $\boldsymbol{F}$ beschaffenen Integralen ist, die zu verschiedenen Exponenten gehören, so ergiebt sich ferner:

VIII. Sind die Wurzeln der Gleichung (2.) von einander verschieden, so enthält jedes Integral ein von Logarithmen freies Glied.

Eine unmittelbare Folgerung aus dem Satze VII. ist der Satz:

IX. Sind die Wurzeln in $(\boldsymbol{R})$ von einander verschieden, so ist der vom Logarithmus freie Theil eines mit Logarithmen behafteten wie $F$ beschaffenen Integrals $\eta$, dessen. Exponent in $(\boldsymbol{R})$ enthalten ist, kein Integral der Differentialgleichung (1.).

Denn ist dieser vom Logarithmus freie Theil $\zeta$, so würde $\eta-\zeta$ zugleich mit $\zeta$ ein Integral der Differentialgleichung (1.) sein; dieses ist aber nach S. VII. nicht möglich, da in $\eta-\zeta$ der vom Logarithmus freie Theil verschwindet.

Eine ähnliche Folgerung für den vom Logarithmus freien Theil eines beliebigen mit Logarithmen behafteten Integrals ergiebt sich aus Satz VIII., wenn die sämmtlichen Wurzeln der Gleichung (2.) von einander verschieden sind.

$\mathrm{X}$. Sind die Wurzeln $r_{1}, r_{2}, \ldots r_{2}$ einer Gruppe $(\boldsymbol{R})$ von einander verschieden, und $v_{1}, v_{2}, \ldots v_{\lambda}$ irgend welche wie $F$ beschaffene Integrale, welche resp. zu diesen Wurzeln als zu ihren Exponenten gehören, so ist eine Gleichung:

$$
\text { (10.) } \quad c_{1} v_{1}+c_{2} v_{2}+\ldots+c_{\lambda} v_{\lambda}=0
$$

mit constanten Coefficienten nicht möglich.

Denn sind die Wurzeln $r_{1}, r_{2}, \ldots r_{\lambda}$ wie im Satze IV. geordnet, und multiplicirt man die Gleichung (10.) mit $(x-a)^{-r_{2}}$, so verschwinden für $x=a$ alle Glieder bis auf $(x-a)^{-r_{\lambda}} v_{\lambda}$, da $r_{1}-r_{\lambda}, r_{2}-r_{\lambda}, \ldots r_{\lambda-1}-r_{\lambda}$ positive ganze Zahlen sind. Daher ist $c_{\lambda}=0$, und die Gleichung (10.) geht in eine 
ähnliche Gleichung zwischen $v_{1}, v_{2}, \ldots v_{\lambda-1}$ über. Es wird nun ebenso gezeigt, dass in dieser $c_{\lambda-1}$ verschwindet; u. s. w.

Da ein wie $F$ beschaffenes Integral eine lineare homogene Function mit constanten Coefficienten der einer Wurzelgruppe $(\boldsymbol{R})$ entsprechenden Integrale des Satzes IV. ist, welche den Exponenten, zu dem $F$ gehört, enthält, so kann man nach Satz III. $\$$. 1 und Satz $\boldsymbol{X}$. d. $\$$ in dem Fundamentalsystem des Satzes IV. diese Integralgruppe durch eine beliebige Gruppe gleichvieler wie $\boldsymbol{F}$ beschaffener Integrale ersetzen, deren zugehörige Exponenten resp. mit den Wurzeln in $(\boldsymbol{R})$ identisch sind, vorausgesetzt, dass nicht zwei dieser Wurzeln einander gleich sind.

Aus den Gleichungen

$$
\begin{gathered}
u_{1}=v_{1}, \quad u_{2}=v_{2}=v_{1} \int z_{1} d x, \quad u_{3}=v_{3}=v_{1} \int z_{1} d x \int t_{1} d x, \ldots \\
u_{\lambda}=v_{\lambda}=v_{1} \int z_{1} d x \int t_{1} d x \ldots \int w_{1} d x
\end{gathered}
$$

ergiebt sich analog wie (A. \$. 6 No. 2):

XI. Sind zwei Wurzeln in $(R)$ einander gleich, so ist die dieser Gruppe entsprechende Integralgruppe des Satzes IV. nothwendig mit Logarithmen behaftet.

Eine Integralgruppe dagegen, welche einer nur verschiedene Wurzeln enthaltenden Gruppe $(\boldsymbol{R})$ entspricht, kann von Logarithmen frei sein.

Die Coefficienten der Fundamentalgleichung (A. \$. 3 Gl. (6.)) hangen von den Constanten der Differentialgleichung im Allgemeinen auf transscendente Weise ab. Es ist bemerkenswerth, dass für einen singulären Punkt $a$, in dessen Umgebung die Differentialgleichung die Form der Differentialgleichung (1.) hat, die Fundamentalgleichung durch die Gleichung (2.) ersetzt werden kann, deren Coefficienten auf algebraische, rationale Weise aus den Constanten der Differentialgleichung gebildet sind. Der Zusammenhang zwischen beiden Gleichungen ist derart, dass das 2лifache der Wurzeln der Gleichung (2.) als die Logarithmen der Wurzeln der Fundamentalgleichung angesehen werden können. - Da die Exponenten, zu welchen die Integrale (7.) gehören, aus der Fundamentalgleichung (A. \$. 3 Gl. (6.)) nur bis auf additive ganze Zahlen sich ergeben, und erst aus der Gleichung (2.) vollkommen bestimmt werden, so scheint es zweckmässig, die Gleichung (2.) die determinirende Fundamentalgleichung der Differentialgleichung (1.) zu nennen. 
Es sei

\section{5.}

(1.)

$$
p_{0} \frac{d^{m} y}{d x^{m}}+p_{1} \frac{d^{m-1} y}{d x^{m-1}}+p_{2} \frac{d^{m-2} y}{d x^{m-2}}+\cdots+p_{m} y+q=0
$$

eine nicht homogene lineare Differentialgleichung mit veränderlichen Coefficienten, und es werde zur Abkürzung

$$
p_{0} \frac{d^{m} y}{d x^{m}}+p_{1} \frac{d^{m-1} y}{d x^{m-1}}+p_{2} \frac{d^{m-2} y}{d x^{m-2}}+\cdots+p_{m} y=Y
$$

gesetzt. Differentiirt man die Gleichung (1.), multiplicirt die entstandene Gleichung mit $q$ und subtrahirt von derselben die mit $\frac{d q}{d x}$ multiplicirte Gleichung (1.), so erhält man

$$
\text { (2.) } \quad \frac{d Y}{d x} q-Y \frac{d q}{d x}=0
$$

oder

$$
\text { (3.) } \quad r_{0} \frac{d^{m+1} y}{d x^{m+1}}+r_{1} \frac{d^{m} y}{d x^{m}}+r_{2} \frac{d^{m-1} y}{d x^{m-1}}+\cdots+r_{m+1} y=0,
$$

eine homogene lineare Differentialgleichung der $m+1^{\text {ten }}$ Ordnung, deren Coefficienten bestimmt sind durch die Gleichungen:

$$
\left\{\begin{array}{c}
r_{\mathfrak{a}+1}=\left(\frac{d p_{\mathfrak{a}}}{d x}+p_{\mathfrak{a}+1}-p_{\mathfrak{a}} \frac{d \log q}{d x}\right)(x-a), \quad(\mathfrak{a}=0,1, \ldots m-1), \\
r_{0}=p_{0}(x-a), \quad r_{m+1}=\left(\frac{d p_{m}}{d x}-p_{m} \frac{d \log q}{d x}\right)(x-a) .
\end{array}\right.
$$

Aus der Differentialgleichung (2.) folgt durch Integration:

$$
\text { (5.) } \mathbf{Y}=C q \text {, }
$$

wo $C$ eine willkürliche Constante. - Ist $y$ ein Integral der Differentialgleichung (2.) oder (3.), und ist der ihm entsprechende Werth der Constante $C$ in der Gleichung (5.) gleich Null, so ist $y$ auch ein Integral der Differentialgleichung:

$$
\text { (6.) } \quad Y=p_{0} \frac{d^{m} y}{d x^{m}}+p_{1} \frac{d^{m-1} y}{d x^{m-1}}+\cdots+p_{m} y=0 \text {. }
$$

Ist die dem $y$ entsprechende Constante $C$ von Null verschieden, so ist $-\frac{y}{C}$ ein Integral der Differentialgleichung (1.). Umgekehrt sind die Integrale der letzteren Differentialgleichung Integrale der Differentialgleichung (3.), welche dem Werthe der Constanten $C=-1$ der Gleichung (5.) entsprechen; und die Integrale der Differentialgleichung (6.) sind Integrale der Differentialgleichung (3.), welche dem Werthe der Constanten $C=0$ der Gleichung (5.) entsprechen. Demnach hat man den Satz: 
I. Jedes Integral der Differentialgleichungen (1.) und (6.) ist ein Integral der Differentialgleichung (3.). Umgekehrt ist jedes Integral der Differentialgleichung (3.) entweder ein Integral der Differentialgleichung (6.) oder bis auf einen constanten Factor ein Integral der Differentialgleichung (1.).

Die Untersuchung der nicht homogenen Differentialgleichung (1.) ist hierdurch auf die Untersuchung der beiden homogenen Differentialgleichungen (3.) und (6.) zurückgeführt.

Es seien nunmehr die Coefficienten der Differentialgleichung (6.) in der Umgebung eines singulären Punktes $a$ eindeutig, und die Integrale derselben für $x=a$ nur so unstetig, dass sie mit bestimmten Potenzen von $x-a$ multiplicirt nicht unendlich sind, so ist:

$$
\text { 7.) } \quad p_{0}=(x-a)^{m}, \quad p_{\mathfrak{a}}=(x-a)^{m-\mathfrak{a}} P_{\mathfrak{a}} \quad(\mathfrak{a}=1,2, \ldots m) \text {, }
$$

wo $P_{\mathfrak{a}}$ in der Umgebung von $a$ eindeutig, continuirlich und endlich ist (\$. 3 ). Ist $\frac{d \log q}{d x}$ in der Umgebung von $a$ eindeutig, so sind die Coefficienten der Differentialgleichung (3.) ebenfalls eindeutig. Sollen die Integrale der Differentialgleichung (1.) dieselbe Eigenschaft wie die Integrale der Differentialgleichung (6.) haben, so gehört dieselbe Eigenschaft nach Satz I. auch den Integralen der Differentialgleichung (3.) an. Es ist daher (nach \$. 3):

(8.) $\quad r_{0}=(x-a)^{m+1}, \quad r_{\mathfrak{a}+1}=(x-a)^{m-\mathfrak{a}} \cdot R_{\mathfrak{a}+1} \quad(\mathfrak{a}=0,1, \ldots m)$,

wo $R_{a+1}$ in der Umgebung von $a$ eindeutig, continuirlich und endlich ist. Aus den Gleichungen (4.), (7.), (8.) folgt:

$$
\begin{gathered}
\boldsymbol{R}_{\mathfrak{a}+1}=(x-a)\left[\frac{d P_{\mathfrak{a}}}{d x}-P_{\mathfrak{a}} \frac{d \log q}{d x}\right]+(m-\mathfrak{a}) P_{\mathfrak{a}}+P_{\mathfrak{a}+1} \quad(\mathfrak{a}=0,1, \ldots m-1), \quad P_{0}=1, \\
R_{m+1}=(x-a)\left[\frac{d P_{m}}{d x}-P_{m} \frac{d \log q}{d x}\right] .
\end{gathered}
$$

Insbesondere ist für $\mathfrak{a}=0, R_{1}=-(x-a) \frac{d \log q}{d x}+m+P_{1}$, also $\frac{d \log q}{d x}=\frac{m+P_{1}-R_{1}}{x-a}$. Daher hat $q$ die Gestalt:

$$
\text { (9.) } \quad q=(x-a)^{\mu} \cdot Q \text {, }
$$

wo $Q$ in der Umgebung von $a$ eindeutig, endlich und continuirlich und in $a$ von Null verschieden ist. Setzt $\operatorname{man} \frac{d \log Q}{d x}=s$, so hat man

$$
\left\{\begin{array}{l}
\boldsymbol{R}_{\mathfrak{a}+1}=P_{\mathfrak{a}+1}+(m-\mathfrak{a}-\mu) P_{\mathfrak{a}}+\left(\frac{d P_{\mathfrak{a}}}{d x}-s P_{\mathfrak{a}}\right)(x-a) \quad(\mathfrak{a}=0,1, \ldots m-1) \\
\boldsymbol{R}_{m+1}=-\mu P_{m}+\left(\frac{d P_{m}}{d x}-s P_{m}\right)(x-a) .
\end{array}\right.
$$

Journal für Mathematik Bd. LXVIII. Heft 4. 
Man hat daher den Satz:

II. Sollen die Integrale der Differentialgleichungen (1.) und (6.), in welchen $p_{0}, p_{1}, \ldots p_{m}, \frac{d \log q}{d x}$ in der Umgebung von a eindeutig sind, für $x=a$ nur so unstetig sein, dass sie mit bestimmten Potenzen von $x-a$ multiplicirt nicht mehr unendlich werden, so haben die Integrale der Differentialgleichung (3.) dieselbe Eigenschaft. Die Coefficienten der Differentialgleichungen (1.) und (6.) sind alsdann durch die Gleichungen (7.) und (9.), die Coefficienten der Differentialgleichung (3.) durch die Gleichungen (8.) und (10.) bestimmt. Sind umgekehrt die Coefficienten der Differentialgleichung (1.) durch die Gleichungen (7.) und (9.) bestimmt, so haben die Integrale derselben zugleich mit den Integralen der Differentialgleichungen (6.) und (3.) die Eigenschaft mit bestimmten Potenzen von $x-a$ multiplicirt für $x=a$ nicht unendlich $z u$ werden.

Aus dem Vorhergehenden ergeben sich noch folgende Sätze, welche Verallgemeinerungen von No. 4. und 5. der A. sind:

III. Sind die Coefficienten der Differentialgleichung (1.) nur für eine endliche Anzahl von Punkten $a_{1}, a_{2}, \ldots a_{\ell}$ unstetig, überdiess $p_{1}, p_{1}, p_{2}, \ldots p_{m}$, $\frac{d \log q}{d x}$ überall eindeutig, und sollen die Integrale der Differentialgleichungen (1.) und (6.) mit bestimmten Potenzen von $x-a_{\mathfrak{a}}$ und $x$ multiplicirt resp. für $x=a_{\mathfrak{a}}$ und $x=\infty$ nicht unendlich werden, so ist:

$$
\text { (11.) } \quad\left\{\begin{array}{c}
p_{10}=\psi^{m}, \quad p_{\mathfrak{a}}=\psi^{m-\mathfrak{a}} \cdot F_{\mathfrak{a}(\rho-1)} \quad(\mathfrak{a}=1,2, \ldots m), \\
q=C\left(x-a_{1}\right)^{\mu_{1}}\left(x-a_{2}\right)^{\mu_{2}} \ldots\left(x-a_{\rho}\right)^{\mu_{\rho}},
\end{array}\right.
$$

wo $\psi=\left(x-a_{1}\right)\left(x-a_{2}\right) \ldots\left(x-a_{Q}\right), C, \mu_{1}, \mu_{2}, \ldots \mu_{Q}$ constante Grössen und $F_{n}$ eine ganze Function höchstens von dem Grade n bezeichnet.

Denn da die Integrale der Differentialgleichung (6.) die verlangte Eigenschaft haben sollen, so müssen zunächst nach $\$ .4$ der $\mathbf{A} . p_{0}$ und $p_{\mathfrak{a}}$ die in den Gleichungen (11.) angegebene Form haben. Nach Satz II. haben aber auch die Integrale der Differentialgleichung (3.) die im Satze angegebene Beschaffenheit, daher ist wieder nach $\$ .4$ der $\mathbf{A}$.

$$
r_{1}=\psi^{m} \cdot F_{Q-1}^{\prime}, \quad r_{0}=\psi^{m+1},
$$

wo $F_{\varrho-1}^{\prime}$ eine ganze Function höchstens von dem Grade $\varrho-1$ bezeichnet. Es ist aber

$$
r_{1}=\left[\frac{d p_{0}}{d x}+p_{1}-p_{0} \frac{d \log q}{d x}\right] \psi=\left[m \frac{d \psi}{d x}+F_{\rho-1}-\psi \frac{d \log q}{d x}\right] \psi^{m} .
$$


Also

oder

$$
F_{\varrho-1}^{\prime}=\left[m \frac{d \psi}{d x}+F_{\varrho-1}-\psi \frac{d \log q}{d x}\right]
$$

$$
\text { (12.) } \frac{d \log q}{d x}=\frac{F_{Q-1}-F_{Q-1}^{\prime}}{\psi}+m \frac{d \log \psi}{d x},
$$

woraus die im Satze für $q$ angegebene Form hervorgeht.

Umgekehrt: IV. Jedes Integral der Differentialgleichungen (1.) und (6.), deren Coefficienten durch die Gleichungen (11.) bestimmt sind, hat die Eigenschaft mit bestimmten Potenzen von $x-a_{\mathfrak{a}}$ und $x$ multiplicirt für $x=a_{\mathfrak{a}}$ und $x=\propto$ resp. nicht unendlich $z u$ werden.

Der Nachweis wird geführt, wenn man den Satz II. für jeden der singulären Punkte $a_{1}, a_{2}, \ldots a_{e}$ anwendet, und um ihn auch für $x=\infty$ anwenden zu können, in der Differentialgleichung (1.) mit den durch Gl. (11.) bestimmten Coefficienten $x=\frac{1}{u}$ substituirt, und alsdann den singulären Punkt Null betrachtet.

\section{6.}

Setzt man in dem Ausdrucke

$\boldsymbol{Y}=(x-a)^{m} \frac{d^{m} y}{d x^{m}}+(x-a)^{m-1} P_{1}(x) \frac{d^{m-1} y}{d x^{m-1}}+(x-a)^{m-2} P_{2}(x) \frac{d^{m-2} y}{d x^{m-2}}+\cdots+P_{m}(x) \cdot y$, wo die Grössen $P$ in der Umgebung von $a$ eindeutig, continuirlich und endlich sind, $y=(x-a)^{\varepsilon} f(x)$, wo $\varepsilon$ eine beliebige Grösse und $f(x)$ eine in der Umgebung von $a$ eindeutige, continuirliche und endliche Function von der Beschaffenheit ist, dass $\eta=(x-a)^{\varepsilon} f(x)$ kein Integral der Differentialgleichung $\boldsymbol{Y}=\mathbf{0}$ ist, so lässt sich die resultirende Function auf die Gestalt $(x-a)^{\mu} \cdot \boldsymbol{Q}$ bringen, wo $"$ eine bestimmte Grösse und $Q$ in der Umgebung von $a$ eindeutig, continuirlich und endlich und in $a$ von Null verschieden ist. - Die Differentialgleichung

$$
\text { (1.) } \quad Y-(x-a)^{\mu} \cdot Q=0
$$

hat alsdann das Integral $\eta=(x-a)^{\varepsilon} f(x)$.

I. Es sei $y_{1}, y_{2}, \ldots y_{m}$ ein Fundamentalsystem der Differentialgleichung

$$
\text { (2.) } \quad Y^{\prime}=0
$$

so ist das System der Integrale $\eta, y_{1}, y_{2}, \ldots y_{m}$ ein Fundamentalsystem der Differentialgleichung $m+1^{\text {ter }}$ Ordnung

(3.) $\left\{\begin{array}{c}(x-a)^{m+1} \frac{d^{m+1} y}{d x^{m+1}}+(x-a)^{m} R_{1}(x) \frac{d^{m} y}{d x^{m}}+(x-a)^{m-1} R_{2}(x) \frac{d^{m-1} y}{d x^{m-1}}+\cdots \\ \cdots+R_{m+1}(x) \cdot y=0\end{array}\right.$ 
wo

$$
\left\{\begin{aligned}
R_{\mathfrak{a}+1}(x) & =P_{\mathfrak{a}+1}(x)+(m-\mathfrak{a}-\mu) P_{\mathfrak{a}}(x)+\left[\frac{d P_{\mathfrak{a}}(x)}{d x}-s P_{\mathfrak{a}}(x)\right](x-a)(\mathfrak{a}=0,1, \ldots m-1) \\
R_{m+1} & =-\mu P_{m}(x)+\left[\frac{d P_{m}(x)}{d x}-s P_{m}(x)\right](x-a), \quad s=\frac{d \log Q}{d x} .
\end{aligned}\right.
$$

Denn nach dem Satze I. des vorigen $\$$. ist zunächst jedes der Integrale $\eta, y_{1}, y_{2}, \ldots y_{m}$ ein Integral der Differentialgleichung (3.). Es kann aber eine Gleichung der Form $C \eta+c_{1} y_{1}+c_{2} y_{2}+\cdots+c_{m} y_{m}=0$ nicht bestehen, ohne dass die constanten Coefficienten derselben verschwinden, da $\eta$ kein Integral der Differentialgleichung (2.) und $y_{1}, y_{2}, \ldots y_{m}$ ein Fundamentalsystem derselben sind. -

Von der Differentialgleichung (1.) wollen wir sagen, sie sei der Differentialgleichung (2.) durch die Substitution $y=(x-a)^{\varepsilon} f(x)$ zugeordnet. Die Grösse $\mu$ heisse der Index der Substitution.

Die zum singulären Punkte $a$ gehörige determinirende Fundamentalgleichung der Differentialgleichung (3.) ist:

$$
\sum_{0}^{m} \mathfrak{a}(r-1)(r-2) \ldots(r-m+\mathfrak{a}) \boldsymbol{R}_{\mathfrak{a}}(a)+R_{m+1}(a)=0
$$

wo $R_{0}(a)=1$ zu setzen ist. Diese Gleichung ist nach den Gleichungen (4.) gleichbedeutend mit:

$$
\sum_{0}^{m} r_{\mathfrak{a}}^{\prime}(r-1)(r-2) \ldots(r-m+\mathfrak{a})\left[P_{\mathfrak{a}}(a)+(m-\mathfrak{a}-\mu+1) P_{\mathfrak{a}-1}(a)\right]-\mu P_{m}(a)=0,
$$

wo wieder $P_{0}(a)=1$ zu setzen ist. Diese Gleichung lässt sich umformen in:

$$
(r-\mu)\left[\sum_{\mathfrak{b}}^{m-1} r(r-1)(r-2) \ldots(r-m+\mathfrak{b}+1) P_{\mathfrak{b}}(a)+P_{m}(a)\right]=0 .
$$

Nun aber ist

$$
\text { (7.) } \sum_{0}^{m-1} r(r-1)(r-2) \ldots(r-m+\mathfrak{b}+1) P_{\mathfrak{b}}(a)+P_{m}(a)=0
$$

die determinirende Fundamentalgleichung der Differentialgleichung (2.). Also:

II. Eine Wurzel der determinirenden Fundamentalgleichung der Differentialgleichung (3.) ist gleich $\mu$, die übrigen $m$ Wurzeln genügen der determinirenden Fundamentalgleichung der Differentialgleichung (2.).

Das Resultat der Substitution von $y=(x-a)^{\varepsilon} f(x)$ in $Y$ hat die Form $(\boldsymbol{x}-\boldsymbol{a})^{\varepsilon} \psi(\boldsymbol{x})$, wo $\psi(\boldsymbol{x})$ eine nach positiven ganzzahligen Potenzen von $\boldsymbol{x}-\boldsymbol{a}$ fortschreitende Reihe ist, in welcher der Coefficient von $(x-a)^{0}$ mit der linken Seite der Gleichung (7.) übereinstimmt, wenn man in dieselbe $r=\varepsilon$ setzt. Dieser Coefficient ist Null oder von Null verschieden, je nachdem $\varepsilon$ eine Wurzel der Gleichung (7.) ist oder nicht. Daher: 
III. Ist $\varepsilon$ nicht einer Wurzel der Gleichung (7.) gleich, so ist der Index der Substitution $\mu$ gleich $\varepsilon$; ist dagegen $\varepsilon$ eine Wurzel der Gleichung (7.), so ist der reelle Theil von $\mu$ grösser als der reelle Theil von $\varepsilon$, so dass $\iota-\varepsilon$ eine positive ganze Zahl wird.

Aus Satz I. folgt:

IV. Enthalten die Ausdrücke irgend welcher Integrale der Differentialgleichung (2.) in der Umgebung von a Logarithmen, so giebt es auch Integrale der Differentialgleichung (3.), deren Ausdrücke in der Umgebung von a mit Logarithmen behaftet sind. Und umgekehrt.

Ist $\varepsilon$ eine Wurzel der Gleichung (7.), und vertheilt man nach Vorschrift des Satzes IV. in No. 4 sowohl die Wurzeln der Gleichung (5.) als die der Gleichung (7.) in Gruppen, und ist $(\boldsymbol{R})$ diejenige Gruppe der Wurzeln der letzteren Gleichung, zu welcher $\varepsilon$ gehört, so entspricht dieser die Gruppe (S) der Wurzeln der Gleichung (5.), welcher ausser den in $\boldsymbol{R}$ enthaltenen Wurzeln die Wurzel $\mu$ angehört. Die übrigen Gruppen der Wurzeln der Gleichung (7.) sind mit den übrigen Gruppen der Wurzeln der Gleichung (5.) identisch (nach Satz II.).

Ist der Index der Substitution $\mu$ einer der Wurzeln in $(\boldsymbol{R})$ gleich, so enthält die Gruppe $(S)$ gleiche Wurzeln, daher ist die dieser Gruppe entsprechende nach dem Satze IV. $\$$. 4 gebildete Integralgruppe mit Logarithmen behattet (Satz XI. \$. 4). - Diese Integralgruppe in dem nach Satz IV. $\$$. 4 gebildeten Fundamentalsysteme der Differentialgleichung (3.) lässt sich aber nach Satz III. \$. 1 durch das System $\eta, u_{1}, u_{2}, \ldots u_{\lambda}$ ersetzen, wenn $\eta=\left(x-a_{/}^{\varepsilon} f(x)\right.$ und $u_{1}, u_{2}, \ldots u_{\lambda}$ die der Gruppe $(\boldsymbol{R})$ entsprechenden nach Satz IV. \$. 4 gebildeten Integrale der Differentialgleichung (2.) sind, da zwischen $\eta, u_{1}, u_{2}, \ldots u_{2}$ zu Folge des Satzes I. d. \$. keine lineare homogene Relation mit constanten Coefficienten stattinden kann. Da aber $\eta$ keinen Logarithmus enthält, so sind irgend welche der Grössen $u_{1}, u_{2}, \ldots u_{\lambda}$, d. h. die der Gruppe $(\boldsymbol{R})$ entprechende Integralgruppe der Differentialgleichung (2.) mit Logarithmen behaftet.

Es werde jetzt umgekehrt vorausgesetzt, dass die einer Gruppe $(\boldsymbol{R})$ von Wurzeln der Gleichung (7.) entsprechende nach S. IV. \$. 4 gebildete Integralgruppe der Differentialgleichung (2.) mit Logarithmen behaftet ist. Die Wurzeln dieser Gruppe in der Reihenfolge des Satzes IV. \$. 4 seien $r_{1}, r_{2}, \ldots r_{\lambda}$ und die ihnen entsprechenden Integrale resp. $u_{1}, u_{2}, \ldots u_{\lambda}$. Sind nicht zwei der Wurzeln in $(\boldsymbol{R})$ einander gleich, und ist $u_{\alpha}$ mit Logarithmen behaftet, so ist der vom 
Logarithmus freie Theil desselben $(x-a)^{r_{\alpha}} \varphi_{\alpha 1}$, welcher nach $\$ .4$ S. V. nicht verschwindet, kein Integral der Differentialgleichung (2.) (\$. 4 S. IX.). Ordnet man daher der Differentialgleichung (2.) die Differentialgleichung (3.) durch die Substitution $y=(x-a)^{r_{\alpha}} \varphi_{\alpha 1}$ zu, so ist nach Satz I. $v_{\alpha}=u_{\alpha}-(x-a)^{r_{\alpha}} \varphi_{\alpha 1}$ ein Integral der Differentialgleichung (3.). Da in diesem mit Logarithmen behafteten Integral das vom Logarithmus freie Glied verschwindet, so sind nach S. VII. \$. 4 nothwendig zwei Wurzeln der Gruppe $(S)$ einander gleich, d. h. $\mu$ mit einer der Wurzeln der Gruppe $(\boldsymbol{R})$ übereinstimmend.

Wird der Differentialgleichung (2.) durch die Substitution $y=(x-a)^{r} f(x)$ die Differentialgleichung (3.) zugeordnet, wo $f(x)$ eine nach ganzen positiven Potenzen von $x-a$ fortschreitende Reihe und $r$ ein Glied einer nach $\$ .4$ S. IV. gebildeten Gruppe $(\boldsymbol{R})$ von Wurzeln der Gleichung (7.): $\boldsymbol{r}_{1} ; \boldsymbol{r}_{2}, \ldots \boldsymbol{r}_{\lambda}$ ist, so werde die Differenz $r_{1}-r_{\lambda}$ mit $s$ und die Summe der Glieder von $f(x)$, welche niedrigere Potenzen von $x-a$ als $(x-a)^{s+1}$ enthalten, mit $g(x)$, endlich die Differenz $f(x)-g(x)$ mit $\varphi(x)$ bezeichnet. Das Resultat der Substitution von $(x-a)^{r} \varphi(x)$ in $\boldsymbol{Y}$ liefert nur Potenzen von $x$-a, deren Exponenten einen grösseren reellen Theil haben als $r_{1}$. Ist daher der Index $\mu$ der Substitution $y=(x-a)^{r} f(x)$ einer Wurzel der Gruppe $(R)$ gleich, so ist der Index der Substitution $y=(x-a)^{r} g(x)$ derselben Wurzel gleich; d. h. giebt es überhaupt eine Substitution $y=(x-a)^{r} f(x)$, deren Index mit $r$ Wurzel derselben Gruppe $(\boldsymbol{R})$ ist, so giebt es eine ganze Function $g(x)$ von der Beschaffenheit, dass der Index der Substitution $y=(x-a)^{r} g(x)$ mit $r$ Wurzel derselben Gruppe ist. - Man hat daher den Satz:

V. Es werde der Differentialgleichung (2.) die Differentialgleichung (3.) durch die Substitution $y=(x-a)^{r} f(x)$, in der $f(x)$ eine ganze Function und $r$ eine Wurzel der zur Differentialgleichung (2.) gehörigen determinirenden Fundamentalgleichung (7.) ist, zugeordnet; es werden ferner sowohl die Wurzeln dieser Gleichung als auch die Wurzeln der zur Differentialgleichung (3.) gehörigen determinirenden Fundamentalgleichung (5.) nach S. IV. $\$ .4$ in Gruppen vertheilt. Ist alsdann $(\boldsymbol{R})$ diejenige Gruppe von Wurzeln der Gleichung (7.), welche $r$ enthält, so entspricht dieser die Gruppe $(S)$ der Wurzeln der Gleichung (5.), welcher ausser den in $(\boldsymbol{R})$ enthaltenen Wurzeln der Index der Substitution $\boldsymbol{M}$ als Glied angehört. Die nach Satz IV. \$. 4 gebildete der Gruppe (R) entsprechende Integralgruppe der Differentialgleichung (2.) ist mit Logarithmen behaftet oder nicht behaftet, je nachdem für irgend eine Wurzel 
$r$ der Gruppe $(\boldsymbol{R})$ oder für keine derselben die ganze Function $f(x)$ so bestimmt werden kann, dass die Gruppe (S) gleiche Wurzeln enthält.

In diesem Satze ist die nothwendige und hinreichende Bedingung für das Auftreten von Logarithmen in den Ausdrücken der Integrale in der Umgebung des singulären Punktes $a$ enthalten. Er bildet demnach eine wesentliche Ergänzung zu den Sätzen XI. $\$ .4$ und A. \$. 6, welche nur eine hinreichende aber keine nothwendige Bedingung enthalten.

Es sei wieder

$$
Y=(x-a)^{m} \frac{d^{m} y}{d x^{m}}+(x-a)^{m-1} P_{1}(x) \frac{d^{m-1} y}{d x^{m-1}}+(x-a)^{m-2} P_{2}(x) \frac{d^{m-2} y}{d x^{m-2}}+\cdots+P_{m}(x) \cdot y,
$$

wo $\boldsymbol{P}_{1}(x), \boldsymbol{P}_{2}(x), \ldots \boldsymbol{P}_{m}(x)$ in der Umgebung von $\boldsymbol{a}$ eindeutig, continuirlich und endliche Functionen sind; ferner sei $(R): r_{1}, r_{2}, \ldots r_{\lambda}$ eine nach S. IV. \$. 4 gebildete Gruppe von Wurzeln der zur Differentialgleichung

$$
\text { (1.) } \quad \boldsymbol{Y}=\mathbf{0}
$$

gehörigen determinirenden Fundamentalgleichung:

$$
\sum_{0}^{m-1} r(r-1) \ldots(r-m+\mathfrak{b}+1) P_{\mathfrak{b}}(a)+P_{m}(a)=0 \quad P_{0}(a)=1 .
$$

Wir wollen nunmehr die analytische Bedingung dafür entwickeln, dass für irgend eine Wurzel $r_{\varrho}$, welche der Gruppe $(R)$ angehört, eine ganze Function $f(x)$ bestimmt werden kann, derart dass der Index der Substitution $y=(x-a)^{r_{\varrho}} f(x)$ mit einer Wurzel der Gruppe $(\boldsymbol{R})$ übereinstimmt. -

Zu dem Ende sei die Reihenfolge der Grössen $r_{1}, r_{2}, \ldots r_{\lambda}$ dieselbe wie im Satze IV. $\$ .4$ und $g(x)$ eine beliebige rationale ganze Function $g(x)=c_{1}+c_{1}(x-a)+c_{2}(x-a)^{2}+\cdots+c_{n}(x-a)^{n}$, und man substituire $y=(x-a)^{r} g(x)$ in $Y$, so erhält man

$$
\text { (3.) } \quad Z=(x-a)^{r} \sum_{0}^{n} \varphi(x, k)(x-a)^{k} c_{k},
$$

wo

$$
\varphi(x, k)=\sum_{0}^{m-1}\left(r_{\lambda}+k\right)\left(r_{\lambda}+k-1\right) \ldots\left(r_{\lambda}+k-m+\mathfrak{b}+1\right) P_{\mathfrak{b}}(x)+P_{m}(x), \quad P_{0}(x)=1
$$

gesetzt ist. Die Wurzeln $r_{\lambda-1}, r_{\lambda-2}, \ldots r_{1}$ haben resp. die Werthe $r_{\lambda}+k_{1}$, $r_{\lambda}+k_{2}, \ldots r_{\lambda}+k_{\lambda-1}$, wo $k_{1}, k_{2}, \ldots k_{\lambda-1}$ eine nicht abnehmende Reihe positiver ganzer Zahlen ist. Da nun die Grössen $\varphi(a, k)$ mit der linken Seite der Gleichung (2.) übereinstimmen, wenn man in derselben $r_{\lambda}+k$ an die Stelle 
von $r$ setzt, so hat man die Gleichungen:

(4.) $\varphi(a, 0)=0, \varphi\left(a, k_{1}\right)=0, \varphi\left(a, k_{2}\right)=0, \ldots \varphi\left(a, k_{\lambda-1}\right)=0$, während $\varphi(a, k)$ für jeden positiven Werth von $k$, der nicht mit $k_{1}, k_{2}, \ldots k_{\lambda-1}$ übereinstimmt, von Null verschieden ist. Um $Z$ nach Potenzen von $x-a$ zu entwickeln, setzen wir $\frac{d^{\lambda} \varphi(x, k)}{d x^{\lambda}}=\varphi^{(\lambda)}(x, k), \varphi^{(a-k)}(a, k)=1.2 .3 \ldots(\mathfrak{a}-k)(\mathfrak{a}, k)$ und $A_{\mathfrak{a}}=\sum_{0}^{\mathfrak{a}}(\mathfrak{a}, k) c_{k} ;$ es ist alsdann, weil $A_{0}=\varphi(a, 0)=0$,

$$
\text { (5.) } \quad Z=(x-a)^{r} \lambda \sum_{1}^{\infty} A_{\mathfrak{a}}(x-a)^{\mathfrak{a}} .
$$

Soll es unter den Indices $1,2, \ldots \lambda-1$, wenigstens einen $\alpha$ geben, für den die Bedingungen

(6.) $\quad A_{1}=0, \quad A_{2}=0, \ldots A_{k_{\alpha}-1}=0, A_{k_{\alpha}}$ nicht gleich Null, erfüllt werden, so ist es mit Rücksicht auf die Gleichungen (4.), wie leicht zu sehen, nothwendig und hinreichend, dass wenigstens für einen der Indices $\alpha=1,2, \ldots \lambda-1$ nicht zugleich die Determinanten

\begin{tabular}{c}
\multicolumn{7}{c}{$\mathrm{D}(\mathfrak{b}, \alpha)=$} \\
$\left(\begin{array}{ccccccc}\left(k_{\mathfrak{b}}+1, k_{\mathfrak{b}}\right) & \left(k_{\mathfrak{b}}+1, k_{\mathfrak{b}}+1\right) & 0 & 0 & \ldots & 0 & 0 \\
\left(k_{\mathfrak{b}}+2, k_{\mathfrak{b}}\right) & \left(k_{\mathfrak{b}}+2, k_{\mathfrak{b}}+1\right) & \left(k_{\mathfrak{b}}+2, k_{\mathfrak{b}}+2\right) & 0 & \ldots & 0 & 0 \\
\vdots & \vdots & \vdots & \vdots & \ldots & \vdots & \vdots \\
\left(k_{\mathfrak{b}+1}-1, k_{\mathfrak{b}}\right) & \left(k_{\mathfrak{b}+1}-1, k_{\mathfrak{b}}+1\right) & \left(k_{\mathfrak{b}+1}-1, k_{\mathfrak{b}}+2\right) & \left(k_{\mathfrak{b}+1}-1, k_{\mathfrak{b}}+3\right) & \ldots & \left(k_{\mathfrak{b}+1}-1, k_{\mathfrak{b}+1}-2\right) & \left(k_{\mathfrak{b}+1}-1, k_{\mathfrak{b}+1}-1\right) \\
\left(k_{\alpha}, k_{\mathfrak{b}}\right) & \left(k_{\alpha}, k_{\mathfrak{b}}+1\right) & \left(k_{\alpha}, k_{\mathfrak{b}}+2\right) & \left(k_{\alpha}, k_{\mathfrak{b}}+3\right) & \ldots & \left(k_{\alpha}, k_{\mathfrak{b}+1}-2\right) & \left(k_{\alpha}, k_{\mathfrak{b}+1}-1\right)\end{array} \mid\right.$
\end{tabular}

für $\mathfrak{b}=0,1,2, \ldots \alpha-1$ verschwinden, wo $k_{0}=0 \mathrm{zu}$ setzen ist. - Ist diese Bedingung für einen bestimmten Werth von $\alpha$ zum ersten Male erfüllt, so sind die Grössen $c_{\mathfrak{a}}$ für die Werthe des Index $\mathfrak{a}=0, k_{1}, k_{2}, \ldots k_{\alpha-1}$ willkürlich und von einander unabhängig geblieben. Es wird daher $\alpha$ ganze Functionen $g_{1}(x), g_{1}(x), g_{2}(x), \ldots g_{\alpha-1}(x)$, welche für $x=a$ nicht verschwinden, geben von der Beschaffenheit, dass die Indices der Substitutionen $y=(x-a)^{r} g_{10}(x)$,

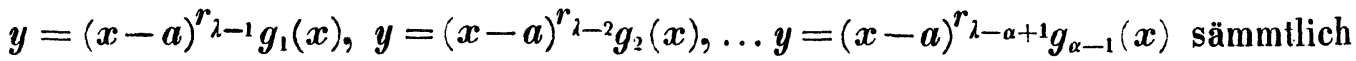
den Werth $r_{\lambda-\alpha}$ haben. - Der Satz V. im vorigen $\$$. lässt sich daher auch durch den folgenden ersetzen:

I. Es seien die Glieder einer nach S. IV. \$. 4 gebildeten Gruppe von Wurzeln der determinirenden Fundamentalgleichung (2.): $r_{\lambda}, r_{\lambda}+k_{1}, r_{\lambda}+k_{2}, \ldots$ $r_{\lambda}+k_{\lambda-1}$, wo $k_{1}, k_{2}, \ldots k_{\lambda-1}$ eine nicht abnehmende Reihe positiver ganzer Zahlen ist, und bildet man die zu dieser Gruppe gehörigen Determinanten $\boldsymbol{D}(\mathfrak{G}, \alpha)$, so ist die nach $\$ .4$ S. IV. der Gruppe $(\boldsymbol{R})$ zugeordnete Integralgruppe 
mit Logarithmen behaftet, oder nicht behaftet, je nachdem für wenigstens einen der Indices $\alpha=1,2, \ldots \lambda-1$ oder keinen derselben in der Reihe der Determinanten $D(0, \alpha), D(1, \alpha), \ldots D(\alpha-1, \alpha)$ sich solche befinden, welche von Null verschieden sind.

Dieser Satz kann auch folgendermassen hergeleitet werden. Setzt man in No. 5 der A. $a_{i}=a$ und $r_{\lambda}$ statt $r_{i l}$, so verschwindet der Coefficient von $c_{k+1}$ in Gleichung $\left(5\right.$.) daselbst, wenn $k$ einen der Werthe $k_{1}-1, k_{2}-1, \ldots k_{\lambda-1}-1$ annimmt. Damit nun, für diese Werthe von $k, c_{k+1}$ endlich bleibe, müssen die zugehörigen Ausdrücke auf der rechten Seite der Gleichung (5.) daselbst verschwinden. Damit ferner Reihenentwickelungen der Gestalt $(x-a)^{r} \sum_{0}^{\infty} \mathfrak{b}_{\mathfrak{a}}(x-a)^{a}$ möglich sind, wenn $r$ eine beliebige der Wurzeln $r_{\lambda}, r_{k}+k_{1}, r_{\lambda}+k_{2}, \ldots r_{\lambda}+k_{\lambda-1}$ und der jedesmalige Werth von $\mathfrak{b}_{v}$ von Null verschieden sein soll, dürfen durch die Gleichungen, welche durch das Verschwinden der rechten Seite der Gleichung (5.) daselbst für die angegebenen Werthe von $k$ erhalten werden, keine Relationen zwischen den Grössen $c_{\mathfrak{a}}$ mit den Indices $\mathfrak{a}=0, k_{1}, k_{2}, \ldots k_{\lambda-1}$ herheigeführt werden. Es lässt sich nun zeigen, dass diesen beiden Bedingungen Genüge geschieht, wenn für jeden Werth von $\alpha=1,2, \ldots \lambda-1$, die Determinanten $D(0, \alpha), D(1, \alpha), \ldots D(\alpha-1, \alpha)$ verschwinden. Tritt dieses ein, so kann man durch ähnliche Schlüsse wie in $\$ .5$ der A. zeigen, dass es zu jedem Werth von $r$ aus der Reihe $r_{\lambda}, r_{\lambda}+k_{1}, r_{\lambda}+k_{2}, \ldots r_{\lambda}+k_{\lambda-1}$ ein Integral der Form $(x-a)^{r} \sum_{10}^{\infty} b_{r a}(x-a)^{a}$ giebt, worin $b_{r 0}$ von Null verschieden ist. Diese Gruppe von Integralen kann man in dem Fundamentalsysteme des Satzes IV. in \$. 4 (nach S.X. in $\$ .4$ und S. III. in \$. 1) der Integralgruppe, welche $(\boldsymbol{R})$ entspricht, substituiren, so dass diese Gruppe nicht mit Logarithmen behaftet ist. -

Die Differenz $r_{1}-r_{l}=\delta$ ist eine ganze Zahl, welche die Ordnung der höchsten in den Determinanten $\boldsymbol{D}(\mathfrak{b}, \alpha)$ auftretenden Ableitung von $\varphi(x, k)$ (für $x=a)$ nicht überschreitet. Jeder Gruppe $(\boldsymbol{R})$ entspricht eine solche Zahl $\delta$. Ist unter allen diesen den verschiedenen Gruppen entsprechenden Zahlen $d$ die grösste, und entwickelt man die Functionen $P_{\mathfrak{a}}(x)$ in Reihen nach Potenzen von $(x-a)$, so treten in keiner der irgend einer Gruppe $(R)$ entsprechenden Determinanten $D(\mathfrak{b}, \alpha)$ die Coefficienten derjenigen Potenzen von $x-a$ auf, deren Exponenten grösser sind als $d$. Diese Coefficienten üben daher auch keinen Einfluss aus auf die Beurtheilung der Frage, ob die irgend einer Gruppe 
(R) zugehörige Integralgruppe mit Logarithmen behaftet ist oder nicht. Man hat daher folgenden Satz:

II. Sind $F_{1}, F_{2}, \ldots F_{n}$ ganze Functionen, welche resp. aus $P_{1}(x)$, $\boldsymbol{P}_{2}(x), \ldots \boldsymbol{P}_{m}(x)$ erhalten werden, wenn man in der Entwickelung derselben nach Potenzen von $x-a$ nur diejenigen Glieder beibehält, welche mit nicht höheren Potenzen von $\dot{x}-a$ als der $d^{\prime e n}$ multiplicirt sind, so sind die irgend einer Gruppe $(\boldsymbol{R})$ entsprechenden Integrale der Differentialgleichung (1.) mit Logarithmen behaftet oder nicht, je nachdem die derselben Gruppe (R) entsprechenden Integrale der Differentialgleichung:

$$
(x-a)^{m} \frac{d^{m} y}{d x^{m}}+F_{1}(x-a)^{m-1} \frac{d^{m-1} y}{d x^{m-1}}+F_{2}(x-a)^{m-2} \frac{d^{m-2} y}{d x^{m-2}}+\cdots+F_{m} y=0
$$

mit Logarithmen behaftet sind oder nicht.

Der Differentialgleichung (7.) gehört die Gleichung (2.) als determinirende Fundamentalgleichung an. Sind die Grössen $P_{\mathfrak{a}}(x)$ gleich constanten Grössen $a_{\mathfrak{a}}$, so dass die Differentialgleichung (1.) die Gestalt:

$$
(x-a)^{m} \frac{d^{m} y}{d x^{m}}+a_{1}(x-a)^{m-1} \frac{d^{m-1} y}{d x^{m-1}}+a_{2}(x-a)^{m-2} \frac{d^{m-2} y}{d x^{m-2}}+\cdots+a_{m} y=0
$$

hat, und die Wurzeln der Gleichung:

$$
\text { (9.) } \quad \sum_{0}^{m-1} r(r-1) \ldots(r-m+\mathfrak{a}+1) a_{\mathfrak{a}}+a_{m}=0 \quad a_{0}=1
$$

verschieden, so sind für jede Gruppe $(\boldsymbol{R})$ die sämmtlichen Determinanten $\boldsymbol{D}(\mathfrak{b}, \alpha)$ gleich Null, da die erste Verticalreihe derselben nur verschwindende Elemente enthält, folglich sind die Integrale nicht mit Logarithmen behaftet (s. A. \$.6).

\section{8.}

Wenn die Coefficienten einer linearen Differentialgleichung

$$
\text { (1.) } \frac{d^{m} y}{d x^{m}}+p_{1} \frac{d^{m-1} y}{d x^{m-1}}+p_{2} \frac{d^{m-2} y}{d x^{m-2}}+\cdots+p_{m} y=0
$$

für einen Werth $x=a$ unendlich werden, übrigens aber in dessen Umgebung eindeutig sind, so hören im Allgemeinen die Integrale der Differentialgleichung auf in der Umgebung dieses Punktes eindeutig, stetig und endlich zu sein. Wenn dieses eintritt, so wollen wir den Punkt $a$ einen wesentlich singulären Punkt der Differentialgleichung (1.) nennen. Wenn aber die Integrale in der Umgebung des Punktes $a$ eindeutig, endlich und continuirlich bleiben, so soll $a$ ein ausserwesentlich singulärer Punkt heissen *).

*) Diese Benennung hat Herr Weierstrass bereits in seiner Vorlesung, welche ich in der A. (Einleitung) angeführt habe, gebraucht. 
Ist $\mathcal{A}_{11}$ die Determinante eines Fundamentalsystems $y_{1}, y_{2}, \ldots y_{m}$ und $\Delta_{\mathfrak{a}}$ diejenige Determinante, welche aus $\Delta_{\mathfrak{1}}$ entsteht, wenn man die $\mathfrak{a}^{\text {te }}$ Vertikalreihe durch $\frac{d^{m} y_{1}}{d x^{m}}, \frac{d^{m} y_{2}}{d x^{m}}, \ldots \frac{d^{m} y_{m}}{d x^{m}}$ ersetzt, so ist ( $\$ .4$ Gl. (7.) der A.)

$$
\text { (2.) } \quad p_{\mathfrak{a}}=-\frac{\Delta_{\mathfrak{a}}}{\Delta_{\mathfrak{1}}} \text {. }
$$

Da nun die Integrale der Differentialgleichung (1.) in der Umgebung eines ausserwesentlich singulären Punktes $a$ eindeutig, continuirlich und endlich sind, so gilt dasselbe für ihre sämmtlichen Ableitungen, folglich auch für die Determinanten $\Delta_{11}$ und $\Delta_{\mathfrak{a}}$. Nun aber sollen für gewisse Werthe von $\mathfrak{a}=1,2, \ldots m$ Coefficienten $p_{\mathfrak{a}}$ für $x=a$ unendlich werden, folglich zeigt die Gleichung (2.), dass $\Delta_{10}$ für $x=a$ verschwindet. Da aber (A. $\$ .4$ Gl. (8.)) $\Delta_{0}$ bis auf einen nicht verschwindenden constanten Factor von der Wahl des Fundamentalsystems unabhängig ist, so folgt:

I. Für einen ausserwesentlich singulären Punkt muss die Determinante jedes Fundamentalsystems verschwinden.

Es seien $b_{1}, b_{1}, \ldots b_{m-1}$ willkürliche Grössen und $x_{0}$ ein nicht singulärer Punkt, so kann man ein Integral $\eta$ der Differentialgleichung (1.) bestimmen, von der Beschaffenheit, dass $b_{0}, b_{1}, b_{2}, \ldots b_{m-1}$ resp. die Werthe von $\eta, \frac{d \eta}{d x}$, $\frac{d^{3} \eta}{d x^{2}}, \ldots \frac{d^{m-1} \eta}{d x^{m-1}}$ für $x=x_{11}$ sind. - Denn man hat das System von Gleichungen:

$$
\left\{\begin{aligned}
\eta & =c_{1} y_{1}+c_{2} y_{2}+\cdots+c_{m} y_{m}, \\
\frac{d \eta}{d x} & =c_{1} \frac{d y_{1}}{d x}+c_{2} \frac{d y_{2}}{d x}+\cdots+c_{m} \frac{d y_{m}}{d x}, \\
\frac{d^{2} \eta}{d x^{2}} & =c_{1} \frac{d^{2} y_{1}}{d x^{2}}+c_{2} \frac{d^{2} y_{2}}{d x^{2}}+\cdots+c_{m} \frac{d^{2} y_{m}}{d x^{2}}, \\
& \vdots \\
\frac{d^{m-1} \eta}{d x^{m-1}} & =c_{1} \frac{d^{m-1} y_{1}}{d x^{m-1}}+c_{2} \frac{d^{m-1} y_{2}}{d x^{m-1}}+\cdots+c_{m} \frac{d^{m-1} y_{m}}{d x^{m-1}},
\end{aligned}\right.
$$

wo $c_{1}, c_{2}, \ldots c_{m}$ Constanten sind. Setzt man rechterhand $x=x_{0}$ und linkerhand statt $\eta, \frac{d \eta}{d x}, \ldots \frac{d^{m-1} \eta}{d x^{m-1}}$ resp. $b_{0}, b_{1}, \ldots b_{m-1}$, so hat man zur Bestimmung der Grössen $c$ ein System linearer Gleichungen, dessen Determinante nicht verschwindet.

Ist dagegen $x_{0}$ ein ausserwesentlich singulärer Punkt, so würde diese Determinante verschwinden, es müssten also die Grössen $b$ eine lineare homogene Relation erfüllen, um resp. als Werthe eines Integrals und dessen $m-1$ ersten Ableitungen für $x=x_{0}$ angesehen werden zu können. 
Das Verschwinden der Determinante eines Fundamentalsystems in den ausserwesentlich singulären Punkten macht also den characteristischen Unterschied zwischen solchen Punkten und den nicht singulären aus.

Da in der Umgebung des ausserwesentlich singulären Punktes $a$ die Coefficienten der Differentialgleichung (1.) eindeutig, die Integrale derselben aber eindeutig, endlich und continuirlich sind, so folgt aus dem Satze in $\mathfrak{\$}$. 3 , dass in der Umgebung von $a$ die Coefficienten die Gestalt:

$$
\text { (4.) } \quad p_{\mathfrak{a}}=\frac{p_{\mathfrak{a}}(x)}{(x-a)^{\mathfrak{a}}}, \quad(\mathfrak{a}=1, \ldots m)
$$

haben, wo $\boldsymbol{P}_{\mathfrak{a}}(x)$ in derselben Umgebung eindeutig, endlich und continuirlich ist. Aus der Gleichung:

$$
\text { (5.) } \quad \Delta_{0}=\text { C. } e^{-\int p_{1} d x}, \quad(\text { A. } \$ .2 \text { Gl. (3.)), }
$$

wo $C$ eine nicht verschwindende Constante bedeutet, und aus Gleichung (4.) folgt :

$$
\text { (6.) } \quad \Delta_{0}=(x-a)^{-P_{1}(a)} \cdot G(x) \text {, }
$$

wo $G(x)$ in der Umgebung von $a$ eindeutig, endlich und continuirlich und in $a$ von Null verschieden ist. Da nun $\Delta_{0}$ in der Umgebung von $a$ eindeutig, endlich und continuirlich und in $a$ gleich Null ist, so ergiebt sich, dass $P_{1}(a)$ eine negative ganze Zahl ist, wenn $a$ einen ausserwesentlich singulären Punkt bedeutet. - Die zum Punkte $a$ gehörige determinirende Fundamentalgleichung der Differentialgleichung (1.), deren Coefficienten durch die Gleichungen (4.) bestimmt werden, ist:

$$
\sum_{0}^{m-1} r(r-1) \ldots(r-m+\mathfrak{a}+1) P_{\mathfrak{a}}(a)+P_{m}(a)=0 \quad P_{0}(a)=1 .
$$

Jeder Wurzel $r$ dieser Gleichung entspricht (nach A. \$.6) ein wie $F$ beschaffenes Integral $y$ derart, dass $(x-a)^{-r} y$ für $x=a$ nicht verschwindet, da aber in der Umgebung eines ausserwesentlich singulären Punktes die Integrale eindeutig, endlich und continuirlich sind, so müssen die Wurzeln der Gleichung (7.) positive ganze Zahlen oder Null sein, daher auch $P_{1}(a), P_{2}(a), \ldots P_{m}(a)$ ganze Zahlen, oder, mit Ausnahme von $P_{1}(a)$, Null. Aus S. XI. $\$ .4$ folgt ferner, dass die Wurzeln derselben Gleichung von einander verschieden sind, es kann daher auch nur eine derselben, folglich nicht gleichzeitig $P_{m}(a)$ und $\sum_{0}^{m-1} 1.2 .3 \ldots(m-\mathfrak{a}-1) P_{\mathfrak{a}}(a)$ verschwinden.

Die Wurzeln der Gleichung (7.) bilden in diesem Falle eine einzige Gruppe. Sind dieselben $r_{1}, r_{2}, \ldots r_{m}$ so geordnet, dass jede folgende kleiner 
ist, als die vorhergehende, und setzt man $r_{m-\mathfrak{a}}=r_{m}+k_{\mathfrak{a}}$, und bildet mit $r_{m}$ statt $r_{\lambda}$ die Determinanten $D(\mathfrak{G}, \alpha)$ der vorigen No., so hat man den Satz:

II. Damit a ein ausserwesentlich singulärer Punkt der Differentialgleichung (1.) sei, ist erforderlich und hinreichend:

1) dass die Differentialgleichung in der Umgebung dieses Punktes die Gestalt:

(8.) $\quad(x-a)^{m} \frac{d^{m} y}{d x^{m}}+P_{1}(x) \cdot(x-a)^{m-1} \frac{d^{m-1} y}{d x^{m-1}}+\cdots+P_{m}(x) \cdot y=0$

habe, wo die Functionen $P_{1}(x), \ldots P_{m}(x)$ in der Umgebung von a eindeutig, continuirlich und endlich sind;

2) dass $P_{1}(a)$ eine negative ganze Zahl ist;

3) dass die Wurzeln der Gleichung (7.) von einander verschieden und, bis auf eine etwa verschwindende, positive ganze Zahlen sind;

4) dass fuir keinen Werth von $\alpha=1,2, \ldots m-1$ irgend eine der Determinanten $D(0, \alpha), D(1, \alpha), \ldots D(\alpha-1, \alpha)$ von Null verschieden ist.

Ist $d=r_{1}-r_{m}$, so kann man nach dem Satze II. des vor. $\$$ bei der Beurtheilung der Art der Singularität des Punktes $a$ in den nach Potenzen von $x-a$ fortschreitenden Reihen $P_{\mathfrak{a}}(x)$ die Glieder mit höheren Potenzen als die $d^{\text {te }}$ unterdrücken, so dass der Punkt $a$ ein wesentlich oder ausserwesentlich singulärer Punkt der Differentialgleichung (8.) ist, je nachdem er ein wesentlich oder ausserwesentlich singulärer Punkt der Differentialgleichung

$$
(x-a)^{m} \frac{d^{m} y}{d x^{m}}+F_{1} \cdot(x-a)^{m-1} \frac{d^{m-1} y}{d x^{m-1}}+\cdots+F_{m} \cdot y=0
$$

ist, wo $F_{1}, F_{2}, \ldots F_{m}$ die durch die Abkürzung von $P_{1}, P_{2}, \ldots P_{m}$ erhaltenen ganzen Functionen sind.

\section{9.}

Wenn die Coefficienten der Differentialgleichung:

$$
(x-a)^{m} \frac{d^{m} y}{d x^{m}}+P_{1}(x) \cdot(x-a)^{m-1} \frac{d^{m-1} y}{d x^{m-1}}+\cdots+P_{m}(x) \cdot y=0
$$

die in S. II. unter No. 1-3 (vor. \$.) angegebenen Bedingungen erfüllen, so können die Ausdrücke der Integrale in der Umgebung des Punktes a (nach §. 4) noch mit Logarithmen behaftet sein. Ob dieses stattfindet oder nicht, lässt sich nach $\$$. 7 beurtheilen, wie dieses in der That im Satze II. vor. \$. unter No. 4 geschehen ist. Diese Frage lässt sich noch auf eine andere Weise entscheiden, wie wir jetzt zeigen wollen. 
Die Integrale der Differentialgleichung (1.) sind unter den gemachten Voraussetzungen wie $F$ beschaffene Functionen, welche zu ganzzahligen positiven Exponenten gehören, nämlich den Wurzeln der Gleichung:

$$
\text { (2.) } \quad \sum_{0}^{m-1} r(r-1) \ldots(r-m+\mathfrak{a}+1) P_{\mathfrak{a}}(a)+P_{m}(a)=0, \quad P_{0}(a)=1 .
$$

Ist $r_{1}$ wieder die grösste Wurzel dieser Gleichung, so sind die Ableitungen eines mit Logarithmen behafteten Integrals spätestens der $r_{1}+1^{\text {ten }}$ Ordnung wie $F$ beschaffene Functionèn, welche zu negativen Exponenten gehören. Dieses ergiebt sich aus der Form des Fundamentalsystems $\$$. 4 S. IV. Dagegen sind die Exponenten, zu welchen die Ableitungen beliebiger Ordnung eines von Logarithmen freien Integrals gehören, stets positiv.

Bildet man aber eine lineare Differentialgleichung für die abhängige Variable $z$ von der Art, dass ihr die $s^{\text {ten }}$ Ableitungen sämmllicher Integrale der Differentialgleichung (1.) und nur diese genügen, so hat dieselbe nach dem Satze in $\$ .3$ die Gestalt:

(3.) $\quad(x-a)^{n} \frac{d^{n} z}{d x^{n}}+Q_{1}(x) \cdot(x-a)^{n-1} \frac{d^{n-1} z}{d x^{n-1}}+\cdots+Q_{n}(x) \cdot z=0$,

wo $Q_{1}(x), \ldots Q_{n}(x)$ in der Umgebung von $a$ eindentig, continuirlich und endlich sind.

Wählt man $s=r_{1}+1$, und ist die determinirende Fundamentalgleichung der Differentialgleichung (3.)

(4.) $\quad \sum_{0}^{n-1} r(r-1) \ldots(r-n+\mathfrak{a}+1) Q_{\mathfrak{a}}(a)+Q_{n}(a)=0, \quad Q_{0}(a)=1$,

I. so ist a ein wesentlich oder ausserwesentlich singulärer Punkt, je nachdem diese Gleichung negative Wurzeln enthält oder nicht.

Es ist daher noch eine Methode anzugeben, wonach eine lineare homogene Differentialgleichung für die abhängige Variabeln z hergestellt wird, der die $\boldsymbol{s}^{\text {ten }}$ Ableitungen sämmtlicher Integrale einer gegebenen Differentialgleichung:

$$
\text { (5.) } \quad p_{0} \frac{d^{m} y}{d x^{m}}+p_{1} \frac{d^{m-1} y}{d x^{m-1}}+\cdots+p_{m} y=0
$$

und nur diese genügen. Eine solche wollen wir nunmehr aufsuchen.

Eliminirt man aus dem Systeme von Gleichungen, welches man erhält, wenn man die Differentialgleichung (5.) mit den durch $s$ aufeinanderfolgende Differentiationen derselben entstandenen verbindet, die Grössen $y, \frac{d y}{d x}, \ldots$ $\frac{d^{6-1} y}{d x^{s-1}}$, so ist das Resultat der Elimination allerdings eine lineare homogene 
Differentialgleichung $m^{\text {ter }}$ Ordnung für $\frac{d^{s} y}{d x^{s}}=z$, und es genügen derselben die $s^{\text {ten }}$ Ableitungen sämmtlicher Integrale der Differentialgleichung (5.). Allein die Ordnung derjenigen Differentialgleichung für $z$, der nur die $s^{\text {ten }}$ Ableitungen der Integrale der Differentialgleichung (5.) genügen, kann niedriger als die $m^{\text {te }}$ sein. In der That hat jedes Integral derselben $z$ die Gestalt:

$$
\text { (6.) } \quad z=c_{1} \frac{d^{s} y_{1}}{d x^{s}}+c_{2} \frac{d^{s} y_{2}}{d x^{s}}+\cdots+c_{m} \frac{d^{s} y_{m}}{d x^{s}},
$$

wo $c_{1}, c_{2}, \ldots c_{m}$ Constanten, und $y_{1}, y_{2}, \ldots y_{m}$ ein Fundamentalsystem der Differentialgleichung (5.) ist. Genügt nun der Differentialgleichung (5.) eine ganze rationale Function $f(x)$, von niedrigerem als dem $\boldsymbol{s}^{\text {ten }}$ Grade, so ist

$$
f(x)=k_{1} y_{1}+k_{2} y_{2}+\cdots+k_{m} y_{m},
$$

wo $k_{1}, k_{2}, \ldots k_{m}$ bestimmte Constanten sind. Aus dieser Gleichung folgt:

$$
\text { (7.) } \quad k_{1} \frac{d^{s} y_{1}}{d x^{s}}+k_{2} \frac{d^{s} y_{2}}{d x^{s}}+\cdots+k_{m} \frac{d^{s} y_{m}}{d x^{s}}=0 \text {. }
$$

Genügen der Differentialgleichung (5.) noch mehrere andere ganze rationale Functionen von niedrigerem Grade als dem $s^{\text {ten }}$, so bestehen noch mehrere der Gleichung (7.) analoge Gleichungen. Es lassen sich alsdann nicht $m$ Integrale $z$ der Form (6.) angeben, zwischen denen keine lineare homogene Relation mit constanten Coefficienten bestände.

Man muss daher einen anderen Weg einschlagen, um diejenige Differentialgleichung herzustellen, welcher die $\boldsymbol{s}^{\text {ten }}$ Ableitungen sämmtlicher Integrale der Differentialgleichung (5.) und nur diese genügen.

Es sei $p_{m-\lambda}$ in der Reihe der Coefficienten der Differentialgleichung (5.) $p_{m}, p_{m-1}, \ldots p_{0}$ der erste, welcher nicht verschwindet, und setzt man $\frac{d^{\lambda} y}{d x^{\lambda}}=u$ und $m-\lambda=\beta$, so geht (5.) über in

$$
\text { (8.) } \quad p_{10} \frac{d^{\beta} u}{d x^{\beta}}+p_{1} \frac{d^{\beta-1} u}{d x^{\beta-1}}+p_{2} \frac{d^{\beta-2} u}{d x^{\beta-2}}+\cdots+p_{\beta} u=0 \text {. }
$$

Ist $y_{1}, y_{2}, \ldots y_{m}$ ein Fundamentalsystem der Differentialgleichung (5.), $u_{1}, u_{2}, \ldots u_{\beta}$ ein Fundamentalsystem der Differentialgleichung (8.), und bezeichnet man das Resultat einer auf eine Function $f(x) \alpha$ mal wiederholte Integration in üblicher Weise mit $\int^{(\alpha)} f(x) d x$, so sind $\int^{(\lambda)} u_{1} d x, \int^{(\lambda)} u_{2} d x, \ldots$ $\int^{(2)} u_{\beta} d x$ Integrale der Differentialgleichung (5.). Es lassen sich daher diese Grössen linear, homogen und mit constanten Coefficienten durch $y_{1}, y_{2}, \ldots y_{m}$ darstellen. Differentiirt man die $\beta$ Gleichungen, welche diese Darstellungen 
liefern, $\lambda$ mal, so folgt, dass $u_{1}, u_{2}, \ldots u_{\beta}$ sich linear, homogen und mit constanten Coefficienten durch die $\lambda^{\text {ten }}$ Ableitungen von $y_{1}, y_{2}, \ldots y_{m}$ darstellen lassen.

II. Der Differentialgleichung (8.) genügen daher die $\lambda^{\text {ten }}$ Ableitungen sämmtlicher Integrale der Differentialgleichung (5.) und nur diese.

Dividirt man Gleichung (8.) durch $p_{\beta}$ und differentiirt, so erhält man eine Gleichung der Form

$$
\text { (9.) } \quad q_{1} \frac{d^{\beta} v}{d x^{\beta}}+q_{1} \frac{d^{\beta-1} v}{d x^{\beta-1}}+\cdots+q_{\beta} v=0,
$$

wenn man $\frac{d u}{d x}=v$ setzt. Sie wird befriedigt durch $v_{1}=\frac{d u_{1}}{d x}, v_{2}=\frac{d u_{2}}{d x}, \ldots v_{\beta}=\frac{d u_{\beta}}{d x}$, wo $u_{1}, u_{2}, \ldots u_{\beta}$ ein Fundamentalsystem der Differentialgleichung (8.) ist. Nun aber ist eine Gleichung der Form $c_{1} v_{1}+c_{2} v_{2}+\cdots+c_{\beta} u_{\beta}=0$, wo $c_{1}, c_{2}, \ldots c_{\beta}$ Constanten sind, unmöglich. Denn aus dieser würde sich ergeben $c_{1} u_{1}+c_{2} u_{2}+\cdots+c_{\beta} u_{\beta}=C$, wo $C$ eine Constante, und zwar eine von Null verschiedene, weil $u_{1}, u_{2}, \ldots u_{\beta}$ Fundamentalsystem von (8.) ist. Es müsste demnach die Differentialgleichung (8.) eine nicht verschwindende Constante zum Integral hahen, was unmöglich ist, weil $p_{\beta}$ von Null verschieden ist. Man hat daher den Satz:

III. Der Differentialgleichung (9.) genügen die ersten Ableitungen sämmtlicher Integrale der Differentialgleichung (8.), und nur diese.

Der Differentialgleichung (9.) genügen also die $\lambda+1^{\text {ten }}$ Ableitungen sämmtlicher Integrale der Differentialgleichung (5.) und nur diese. Daher ergiebt sich folgendes Verfahren, um eine lineare homogene Differentialgleichung herzustellen, welcher die $s^{\text {ten }}$ Ableitungen sämmtlicher Integrale der Differentialgleichung (5.) und nur diese genügen:

Man dividire die Differentialgleichung (5.) durch den Coefficienten der niedrigsten der wirklich darin enthaltenen Ableitungen, abgesehen von einem constanten Factor, und differentiire die so erhaltene Gleichung. Auf die alsdann entstandene Differentialgleichung wende man dasselbe Verfahren an, auf die nunmehr entstandene wiederum dasselbe Verfahren, und fahre nur so lange fort, bis man zum ersten Male eine Differentialgleichung erhäl, in welcher die niedrigste der wirklich darin enthaltenen Differentialquotienten gleicher oder höherer Ordnung als $\frac{d^{s} y}{d x^{s}}$ ist, so ist diese Differentialgleichung die gesuchte. -

Es ist noch zu bemerken, dass in dem Systeme von Differentialgleichungen, welches man erhält, wenn man $s=r_{1}+1$ annimmt und dieses Verfahren auf die Differentialgleichung (1.) anwendet, jede Differentialgleichung 
als Differentialgleichung für die niedrigste der wirklich darin enthaltenen $\mathbf{A b}$ leitungen aufgefasst, die Gestalt der Differentialgleichung (3.) hat, wenn man in dieser statt $y$ diese niedrigste Ableitung setzt. Die derselben zugehörige determinirende Fundamentalgleichung (4.) darf keine negativen Wurzeln enthalten, wenn $a$ ein ausserwesentlich singulärer Punkt der Differentialgleichung (1.) ist. Zieht man es daher vor, successive die einzelnen den verschiedenen Differentialgleichungen des Systems angehörigen Fundamentalgleichungen zu untersuchen, so wird man häufig nicht erst bis zur Differentialgleichung für $\frac{d^{r_{1}+1} y}{d x^{r_{1}+1}}$ vorzuschreiten brauchen, um zu entscheiden, ob $a$ ein wesentlich oder ausserwesentlich singulärer Punkt der Differentialgleichung (1.) ist, nämlich dann, wenn eine der erwähnten Fundamentalgleichungen negative Wurzeln enthält. -

Berlin, im Januar 1868. 